\title{
C-C Bond Formation by Sequential Intramolecular Hydrogen Atom Transfer/ Intermolecular Radical Allylation Reaction in Carbohydrate Systems
}

\author{
Elisa I. León, ${ }^{[a]}$ Ángeles Martín,*[a] Inés Pérez-Martín,*[a] Luís M. Quintanal, ${ }^{[a]}$ and \\ Ernesto Suárez ${ }^{[a]}$
}

Keywords: Carbohydrates / Radical reactions / Hydrogen transfer / Radical allylation / C-C coupling

A tandem 1,5-, 1,6- or 1,8-intramolecular hydrogen atom transfer (HAT)/intermolecular radical allylation using monoor $(1<4)-O$-disaccharide models is described. It is a simple, sequential, stereocontrolled methodology for $\mathrm{C}-\mathrm{C}$ bond for- mation by remote free radical functionalization, giving access to interesting carbohydrate structures carrying differently functionalized tethers.

\section{Introduction}

The use of radical methods in carbohydrate chemistry has been recently and thoroughly reviewed..$^{[1]}$ Furthermore, a number of reviews have arisen where the main topic focuses on inter- and intramolecular carbon-carbon bondforming radical reactions, with emphasis placed on the preparation of $C$-glycosides, $C$-ketosides, $C$-disaccharides, branched-chain sugars, and carbocycles. ${ }^{[2-7]}$ However, the regioselective generation of $\mathrm{C}$-radicals in specific nonactivated positions of the sugar skeleton is not an easy task unless it is conducted intramolecularly. In this sense, remote free radical functionalization, especially carbon-carbon bond formation, on unactivated carbon atoms by intramolecular hydrogen atom transfer (HAT) reactions might be a valuable methodology ${ }_{.}^{[8]}$ The transposition of a radical centre from an oxygen atom to a remote nonactivated carbon atom offers possibilities for the introduction of different functional groups, ${ }^{[1,9]}$ or to form new $\mathrm{C}-\mathrm{C}$ bonds otherwise difficult to achieve, particularly in carbohydrate structures. ${ }^{[10,11]}$ However, despite its potential usefulness, this approach has not been widely employed, probably because of the difficulties encountered in the preparation of alkoxyl radicals under nonoxidative conditions. ${ }^{[12]}$ Related to this, we have recently envisaged a straightforward methodology for the preparation of $C$-ketosides using an intramolecular HAT reaction under reductive conditions as the key step in elaborating monosaccharide systems. ${ }^{[10 a]}$ A conveniently disposed alkoxyl radical generated from a phthalimide supported on a three-carbon chain of a $C$-glycoside, would trigger the intramolecular HAT reaction at the pseudo-

[a] Instituto de Productos Naturales y Agrobioogía del C.S.I.C Carretera de la Esperanza 3, 38206 La Laguna, Tenerife, Spain

Fax: +34-922-260-135

E-mail: angelesmartin@ipna.csic.es ines@ipna.csic.es

Supporting information for this article is available on the WWW under http://dx.doi.org/10.1002/ejoc.201200300. anomeric centre, and the resulting C-radical intermediate could be added intermolecularly to allylstannane to give the corresponding $C$-ketoside as depicted in Scheme 1. The stereochemistry of the quaternary carbon atom, carrying two differently functionalized carbon tethers, is stereoelectronically controlled and independent of that of the starting isomer.
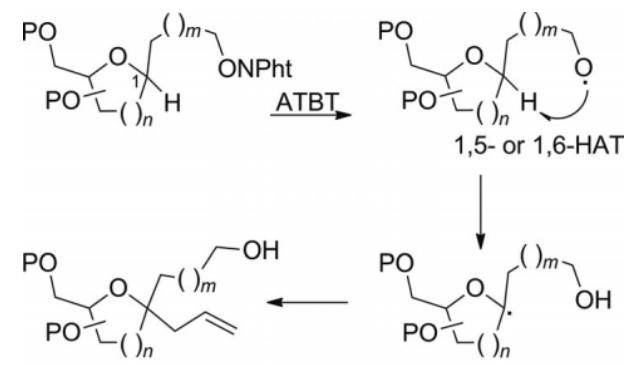

ATBT $=$ Allyltri- $n$-butyltin

Scheme 1. Synthesis of $C$-ketosides by sequential HAT/radical allylation. ATBT $=$ allyltri- $n$-butyltin. AIBN $=$ azobis(isobutyronitrile).

\section{Results and Discussion}

We reasoned that our previous work would be incomplete if we did not perform a similar study starting from $O$ glycosides, analogous to the $C$-glycosides employed before. ${ }^{[10 a]}$ We did similar work a few years ago with the radical study of the conformational differences between $C$ - and $O$-glycosides when treated with tri- $n$-butyltin hydride (TBTH)/AIBN. ${ }^{[13]}$ In order to acquire additional insight into the HAT reaction mechanism, we decided to investigate the reaction course of the sequential intramolecular HAT/intermolecular allylation in $O$-glycosides. For this 
purpose, we have used the fragmentation of $N$-hydroxyphthalimide derivatives to generate the alkoxyl radicals and allyltri- $n$-butyltin (ATBT) as a radical trap. The synthesis of the $O$-phthalimido derivatives was readily achieved from the corresponding alcohol and $N$-hydroxyphthalimide under Mitsunobu conditions in accordance with a previously described protocol. ${ }^{[14]}$ In our earlier work with $C$-glycosides, it was observed that when phthalimide $\mathbf{1}$ was treated with ATBT/AIBN, product $\mathbf{2}$ was obtained resulting from the regio- and stereoselective functionalization at $\mathrm{C}-1$ (Scheme 2). ${ }^{[10 \mathrm{a}]}$ However, the thermally initiated reaction of phthalimide $\mathbf{3}$ with ATBT/AIBN gave $\mathbf{4}$ in $48 \%$ yield as a mixture of diastereomers (inseparable by ordinary column chromatography) and $10 \%$ of prematurely reduced alcohol (Scheme 2; Table 1, Entry 1). Product 4 resulted from the intramolecular 1,5-HAT at C-1, giving a C-radical sufficiently stable to undergo a subsequent $\beta$-fragmentation to generate a C-5 radical, which was finally trapped by ATBT.

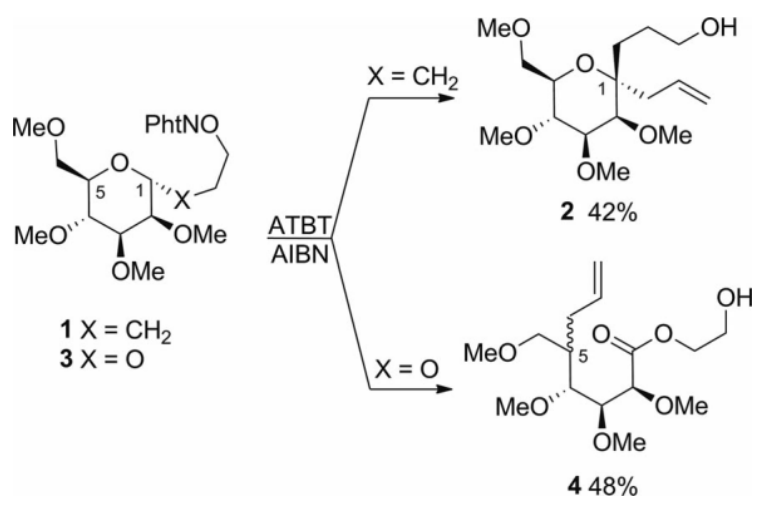

Scheme 2. Sequential HAT/radical allylations of $C$ - and $O$-glycosides derived from d-mannopyranose with four-atom tethers. ATBT $=$ allyltri- $n$-butyltin. AIBN = azobis(isobutyronitrile).

Curiously, although the resulting products came from HAT at C-1, no products derived from radical quenching at this carbon atom were obtained, as was observed for the $C$-glycoside counterpart 1 (Scheme 2). Probably, this disparity between $C$ - and $O$-glycosides was due to the presence of the exo-anomeric effect in the $O$-glycoside precursors, which renders the $\mathrm{C}-1$ radical stable enough to promote the intramolecular $\beta$-fragmentation reaction of the $\mathrm{C} 5-\mathrm{O}$ bond before being trapped by the allylstannane. At the same time, possible electronic interactions between the nonbonding electron pairs of the $O$-glycoside fragment and the stannylated radical intermediates could support the radical fragmentation to the detriment of the allyl trapping at C-1.

We also synthesized tetraacetate 5. The presence of such electron-withdrawing groups (EWGs) should deactivate the hydrogen atoms for abstraction by the electrophilic alkoxyl radicals (Table 1, Entry 2). ${ }^{[15]}$ In fact, only product 6, originating from direct allylation of the O-radical intermediate, was obtained in low yield, and no product derived from HAT was observed, confirming such a deactivation. Subsequently, the reaction was also extended to isomer 10, where the additional carbon atom in the tether would require a less-favoured HAT through a seven-membered tran-
Table 1. Intramolecular HAT/intermolecular radical allylation of $O$-phthalimido- $O$-glycosides.

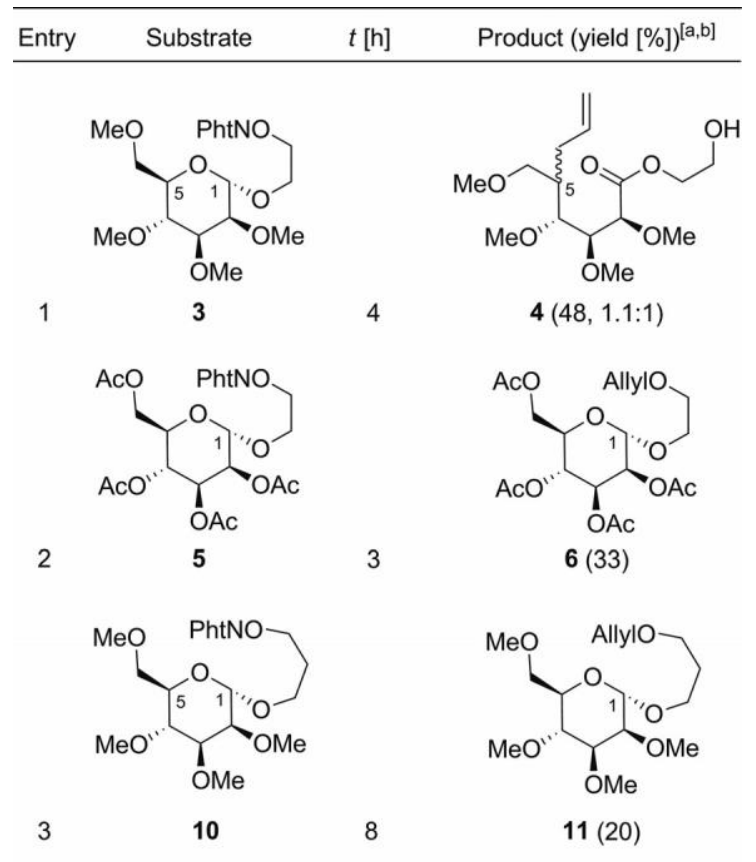

[a] The phthalimide precursor $(1 \mathrm{mmol})$ in dry benzene $(10 \mathrm{~mL})$ containing ATBT $(10 \mathrm{mmol})$ was refluxed, and AIBN (0.01 mmol) was added every hour until the starting material was completely consumed. [b] Previously reduced alcohol was also obtained in 10 $39 \%$ yield.

sition state (TS). The reaction with the C-counterpart 7 happened fairly well and gave $\mathbf{8}$ in $35 \%$ yield (Scheme $3) .{ }^{[10 a]}$ Unfortunately, direct allylation of the $\mathrm{O}$ - radical intermediate derived from $\mathbf{1 0}$ occurred, giving $\mathbf{1 1}$ in $20 \%$ yield together with the prematurely reduced alcohol in 39 $\%$ yield (Table 1, Entry 3 ). A possible explanation could be the destabilizing electronic interactions between the allylstannyl radical intermediates and the nonbonding electron pairs of the oxygen atom in the glycosidic tether, which in some way could disfavour the HAT at C-1, directing the reaction to the premature capture of the starting $\mathrm{O}$-radical. It is interesting to note that when the same starting phthalimides $\mathbf{7}$ and $\mathbf{1 0}$ were treated with the sterically less hindered tri- $n$-butyltin hydride (TBTH) different results were obtained. Radical abstraction in the intermediate derived from 7 was successfully and exclusively achieved at C-1 giving 9 by a 1,6-HAT for the more flexible $C$-glycoside. On the other hand, radical abstraction in the intermediate derived from 10 occurred mainly at C-5 by a 1,8-HAT, affording products $\mathbf{1 3}$ in $27 \%$ yield, together with a $13 \%$ yield of the C-1-inverted derivative 12 (Scheme 3). ${ }^{[13]}$ In our opinion, this disparity in selectivity was probably caused by the different steric natures of the corresponding stannylated radical intermediates resulting from TBTH and ATBT treatments.

Next, we studied the HAT of the $\alpha$ - and $\beta$-isomers of $d$ glucose derivatives $\mathbf{1 4}$ and 17. We observed that in these cases, an inseparable mixture of epimers $\mathbf{1 6}$ was mainly ob- 

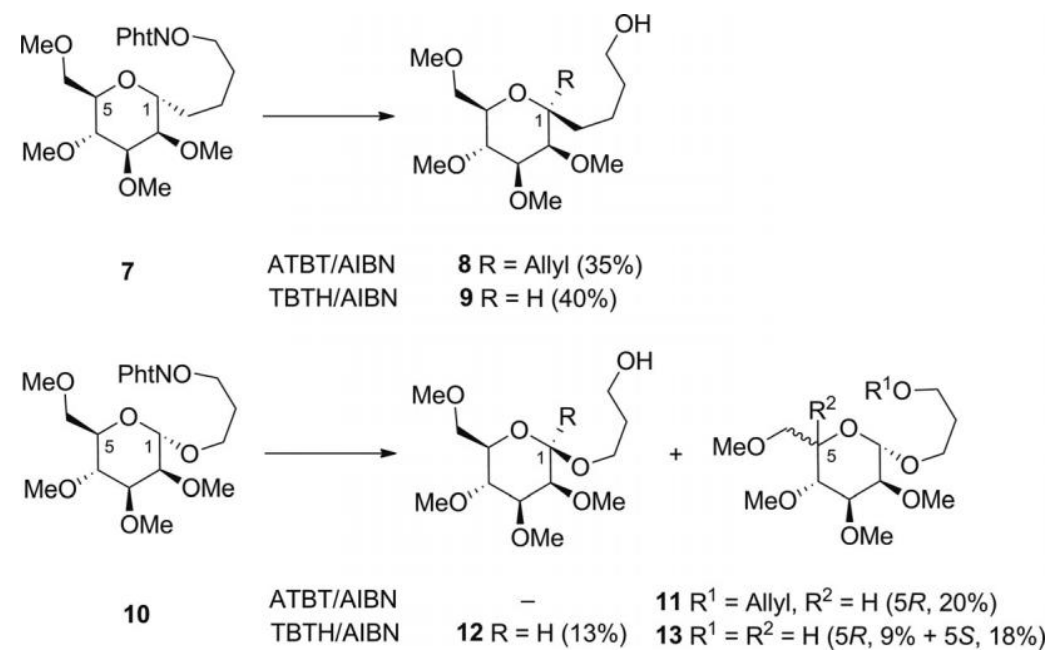

Scheme 3. Sequential 1,6-HAT/radical allylation of $C$ - and $O$-glycosides derived from d-mannopyranose with five-atom tethers. TBTH $=$ tri- $n$-butyltin hydride.

Table 2. Intramolecular HAT/intermolecular radical allylation of $O$-phthalimido- $O$-glycosides.

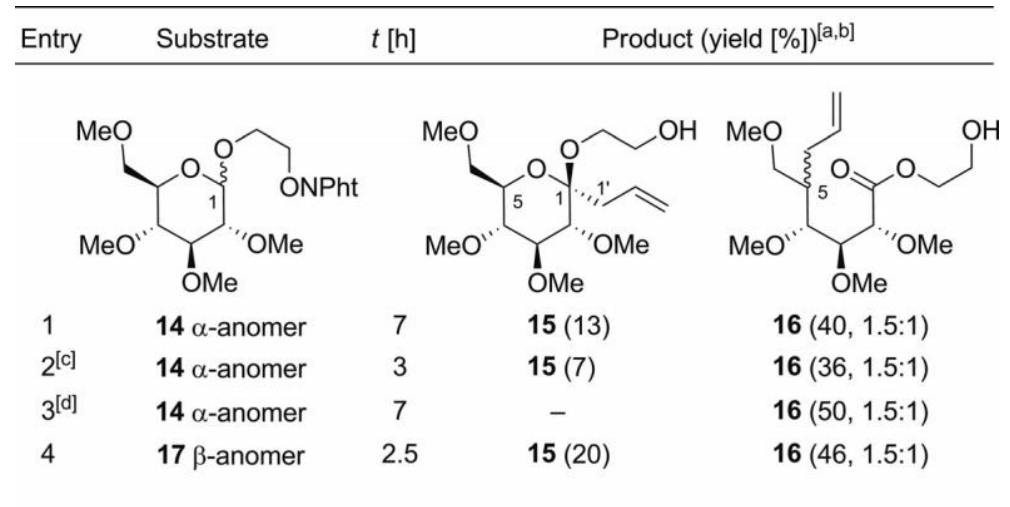

[a] The phthalimide precursor $(1 \mathrm{mmol})$ in dry benzene $(10 \mathrm{~mL})$ containing ATBT $(10 \mathrm{mmol})$ was refluxed, and AIBN (0.01 mmol) was added every hour until the starting material was consumed. [b] Previously reduced alcohol was also obtained in 3-10\% yield. [c] 5 equiv. of ATBT and $10 \mathrm{~mL} / \mathrm{mmol} \mathrm{PhH}$ were employed. [d] 5 equiv. of ATBT and $30 \mathrm{~mL} / \mathrm{mmol} \mathrm{PhH}$ were employed.

tained, derived from the sequential 1,5 -HAT at C-1, $\beta$-fragmentation and radical allyl trapping at C-5 (Table 2, Entries 1-4), as was previously observed in Entry 1, Table 1. However, in these cases, product $\mathbf{1 5}$, resulting from 1,5 HAT at C-1 and subsequent radical allylation was also achieved as expected, together with a small amount of prematurely reduced alcohol. It is interesting to note that regardless of the stereochemistry at the anomeric centre of the phthalimide precursors ( $\alpha$ - or $\beta$-isomer), the $\mathrm{C}$-radical quenching occurred stereoselectively along the axial direction, consistent with previous studies. ${ }^{[16]}$ Since electrophilic radicals abstract axial hydrogen atoms much faster than the equatorial ones, it is not surprising that the reaction of the $\beta$-isomer 17 gave somewhat better results (Table 2, En- try 4). ${ }^{[17]}$

Additionally, dilution experiments were made with precursor 14 in order to analyze the influence of concentration over the course of the radical reaction (Table 2, Entries 2 and 3). It was observed that smaller amounts of ATBT, such as 5 equiv. instead of 10 , gave much more $\beta$-fragmented product 16 relative to 1 -allyl product 15 or prematurely reduced alcohol (Table 2, Entry 2). Moreover, when the benzene dilution was increased to $30 \mathrm{~mL} / \mathrm{mmol}$ and 5 equiv. of ATBT was added, only $\beta$-fragmented product 16 was obtained in $50 \%$ yield, which shows that stabilization of the $\mathrm{C}-1$ radical was enhanced, favouring the intramolecular rupture of the $\mathrm{C} 5-\mathrm{O}$ bond before being trapped by the allylstannane (Table 2, Entry 3). Reactions with dilutions $>30 \mathrm{~mL} / \mathrm{mmol}$ did not enhance the yield of the fragmented product.

We next focused our attention on applying this sequential methodology to functionalize specific $(1<4)$ - $O$-disaccharide systems that fulfill the stereochemical and conformational requirements [i.e., with the correct relative stereochemistry at the four chiral centers (C-4, C-5, C-1' and C$\left.5^{\prime}\right)$ ] to carry out long-distance HAT exclusively at C-5' 
through a nine-membered transition state. ${ }^{[9 a, 18]}$ Accordingly, switching the $(1<4)-O$-disaccharide structure with the requisites mentioned before (Scheme 4).
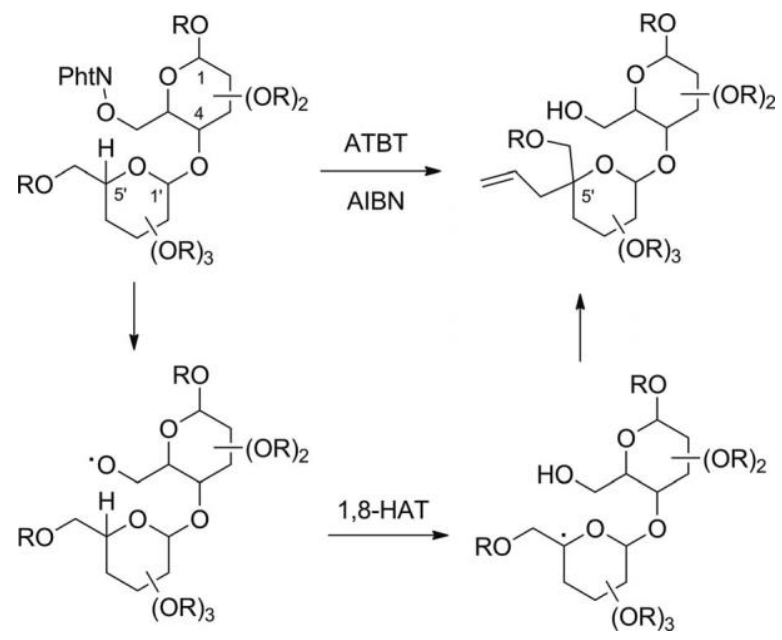

Scheme 4. Sequential intramolecular 1,8-HAT/intermolecular radical allylation of $O$-phthalimide precursors in $(1<4)$ - $O$-disaccharides.

First, we prepared a pair of $\alpha-1-$ Rhap- $(1<4)-\alpha-\mathrm{d}-\mathrm{Gal} p$ disaccharides, which gave good results with TBTH/AIBN treatment. ${ }^{[18]}$ Phthalimides $\mathbf{1 8}$ and $\mathbf{2 1}$ were successfully synthesized and submitted to the intramolecular HAT/intermolecular radical allylation reaction, with ATBT and AIBN in dry benzene at reflux (Table 3, Entries 1 and 2). Precursor 18 gave a mixture of two compounds 19 and 20, with a global yield of $87 \%$ as a result of the 1,8-HAT reaction. The major product $\mathbf{1 9}$ was derived from the intramolecular 1,8-HAT to give a C-5' radical intermediate, which was intermolecularly trapped by allylstannane. This compound was obtained as a single diastereoisomer within the detection limits of ${ }^{1} \mathrm{H}$ and ${ }^{13} \mathrm{C}$ NMR spectroscopy, by the analysis of the crude reaction mixtures. The stereochemistry at the quaternary centre was tentatively assigned as $(R)$ on the basis of the NOE interaction observed between $4^{\prime}-\mathrm{H}$ and 6 '-Me, showing that the radical quenching took place axially as expected. ${ }^{[19]}$ On the other hand, the minor product 20, which involves a 4'-deoxygenated carbon atom and a 1,3,5-trioxocane ring as principal features, could be obtained by a plausible mechanism, which consists of a $5^{\prime}-$ radical reductive elimination to give a 4 '-enol ether and subsequent alcohol addition. Interestingly, the oxygen atom at C-6 acted with an umpolung reactivity during the reaction, first as an electrophilic alkoxyl radical and later as a nucleophile.

Acetylated derivative $\mathbf{2 1}$ also gave the expected allyl product 22 in $42 \%$ yield as a single diastereomer with a configuration at $\mathrm{C}-5^{\prime}$ determined as $(R)$ on the basis of the NOE interaction observed between $4^{\prime}-\mathrm{H}$ and $6^{\prime}-\mathrm{Me}$ (Table 3, Entry 2). Moreover, three other minor products, 23, 24 and prematurely reduced alcohol, were also obtained in $11 \%, 10 \%$ and $14 \%$ yields, respectively. None of the minor products resulted from the 1,8-HAT, which suggests that the electron-withdrawing acetyl group at C-4' could somehow inhibit hydrogen abstraction at C-5'. O-Allyl derivative $\mathbf{2 3}$ came from the early quenching of the O-radical by allylstannane, while $\mathbf{2 4}$ probably resulted from the radical reduction with ATBT of a feasible aldehyde intermediate. $^{[20]}$ The stereochemistry at C-6 was tentatively established as $(S)$ according to the guidelines of the Felkin-Anh rule for radicals. ${ }^{[21]}$

To better understand the scope and stereoselectivity of this C-5' radical stannane quenching, we prepared $\alpha$-dTal $p$ - $(1<4)-\alpha-d-G l c p 25$ (Table 3, Entry 3). In this case 26 was obtained in $53 \%$ yield as an inseparable (by ordinary column chromatography) mixture of epimers at C-5', together with the corresponding prematurely reduced alcohol in $10 \%$ yield. Possibly, the presence of the three axial substituents of the $\alpha$-d-talose ring could trigger a modest chair inversion, yielding a small amount of $\left(5^{\prime} R\right)$ derivative, which would result, as expected, from the axial quenching.

Next, acetylated $\alpha$-d-Glc $p-(1<4)-\beta-\mathrm{d}-\mathrm{Glc} p$ derivative $(\beta$ maltose) 27 was submitted to the radical sequence. Unfor-

tunately, no products were obtained from the HAT, but rather from $\beta$-fragmentation followed by allylation (Table 3, Entry 4), in contrast to the good results observed with the TBTH/AIBN treatment. ${ }^{[18 \mathrm{~b}]}$ Products 28 were generated in $68 \%$ yield as an inseparable mixture of diastereomers, together with an $11 \%$ yield of the prematurely reduced alcohol. The stereochemistry at C-5 of the major product was tentatively established as $(R)$ according to the NOE correlation observed between $5-\mathrm{H}$ and $1-$ and $3-\mathrm{H}$. Similarly, methylated derivative $\mathbf{2 9}$ also gave $\mathbf{3 0}$ as a mixture of epimers in $35 \%$ yield, in which NOE interactions were observed for the major isomer $(5 R)$ between $5-\mathrm{H}$ and $3-\mathrm{H}$.

The corresponding prematurely reduced alcohol was also produced in $9 \%$ yield (Table 3, Entry 5). The main product consisted of the homoallylic alcohol $\mathbf{3 1}$ which, as with product 24 (Table 3, Entry 2), probably resulted from the radical reduction with ATBT of a feasible aldehyde intermediate.

As previously, the stereochemistry at C-6 was tentatively assigned as $(S)$ according to the Felkin-Anh rule for radicals. ${ }^{[21]}$ This result is consistent with that obtained for $\mathrm{d}$ mannose derivative $\mathbf{1 0}$ (Table 1, Entry 3), where the expected 1,8-HAT was also not observed when treated with ATBT; probably due to the hindrance and instability of the supposed allylated stannyl intermediate resulting from the HAT.

Next, the question arose as to whether the substituent at C-5' in the hexopyranose disaccharide models could somehow hinder the intramolecular abstraction and subsequent allylation and thereby favour other radical processes. The absence of such a substituent would reduce the steric hindrance at $\mathrm{C}-5^{\prime}$. At the same time, this would be detrimental to the stability of the corresponding C-radical, because a secondary C-5'-radical would be generated instead of a tertiary one. To test this, we prepared $\alpha$-d-Lyx $p$ - $(1<4)-\alpha$-dGlcp 32, a pentopyranose derivative with no substitution at C-5', and submitted it to the HAT/radical allylation conditions (Table 4, Entry 1). An inseparable mixture of allyl epi- 
Table 3. Intramolecular 1,8-HAT/intermolecular radical allylation of $O$-phthalimido- $(1<4)$ - $O$-disaccharides.

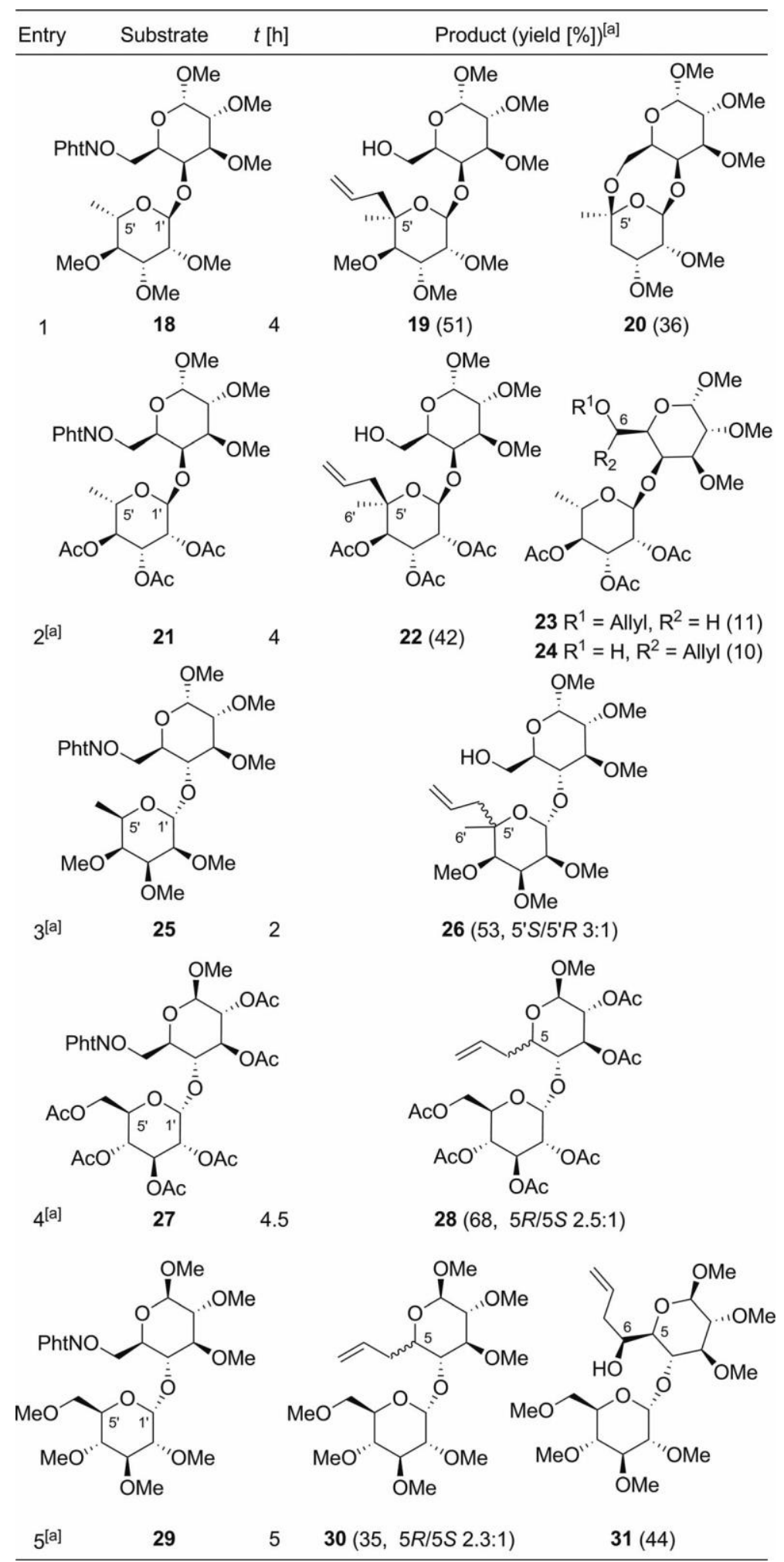

[a] Previously reduced alcohol was also obtained in $10-14 \%$ yield.

mers at C-5', 33, was obtained in $29 \%$ yield together with the $O$-allyl derivative 34 in $17 \%$ yield (resulting from the early quenching of the O-radical by allylstannane) and the prematurely reduced alcohol generated in $29 \%$ yield. In view of this result, it appears that the presence of any group at C-5' apparently does not have a crucial effect on HAT, because C-5'-allyl product $\mathbf{3 3}$ was produced, although in lower yield. 


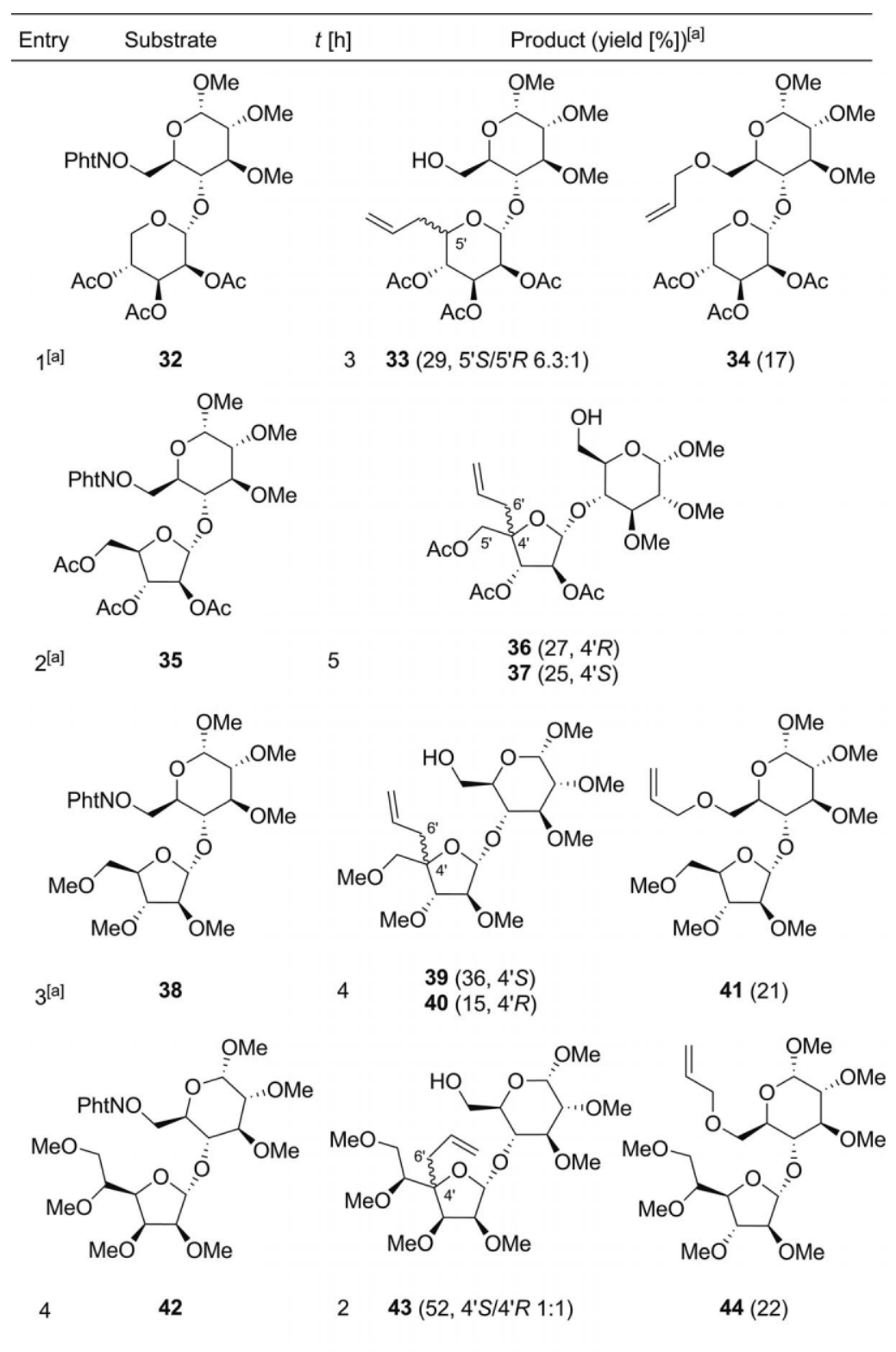

[a] Previously reduced alcohol was also obtained in $12-29 \%$ yield.

Until now, only pento- and hexopyranose models have been researched. Hence we thought that to complete the study, pento- and hexofuranose models should be also examined where, unlike pyranosyl radicals, steric effects seem to control the stereoselectivity. ${ }^{[10 a, 22,23]}$ We prepared the acetylated pentofuranose $\alpha$-d-Araf-(1<4)- $\alpha$-d-Glc $p \quad 35$, which was submitted to the HAT/allylation standard conditions, giving rise to $\mathrm{C}-4$ '-allyl derivatives $\mathbf{3 6}$ and $\mathbf{3 7}$ in $52 \%$ global yield, together with the corresponding prematurely reduced alcohol in $12 \%$ (Table 4, Entry 2). The stereochemistry at the quaternary centre was established by the NOE correlation observed between $6^{\prime}-\mathrm{H}$ and $2^{\prime}-\mathrm{H}$ in $\mathbf{3 6}$ and between $6{ }^{\prime}-\mathrm{H}$ and $3^{\prime}-\mathrm{H}$ in $\mathbf{3 7}$. Almost the same result was obtained for the methylated phthalimide 38, which also gave the C-4'-allyl products $\mathbf{3 9}$ and $\mathbf{4 0}$ in $51 \%$ global yield. The stereochemistry at C-4' was well defined by NOE interactions between $6^{\prime}-\mathrm{H}$ and $3^{\prime}-\mathrm{H}$ for 39 and $5^{\prime}-\mathrm{H}$ and $1^{\prime}-\mathrm{H}$ for 40, confirming that this time the allylation took place slightly more favourably on the less hindered $\beta$-face. $O$-Allyl product 41 was also generated in $21 \%$ yield, together with the previously reduced alcohol in $22 \%$ yield (Table 4, Entry 3$)$.

Finally, the hexofuranose $\alpha$-d-Manf-(1<4)- $\alpha$-d-Glcp 42 was studied, giving as expected an inseparable mixture of allyl epimers $\mathbf{4 3}$ in $52 \%$ yield together with $O$-allyl derivative 44 obtained in $22 \%$ yield (Table 4, Entry 4). 
This procedure provides a simple stereocontrolled methodology for $\mathrm{C}-\mathrm{C}$ bond formation by a remote free radical functionalization. The resulting interesting carbohydrate structures may be useful as scaffolds and synthetic building blocks. To the best of our knowledge, no precedents exist for this tandem intramolecular HAT/intermolecular allylation reaction, with the exception of our preliminary report on $C$-glycosides. In this present work, we have extended this methodology to more complex $O$-glycosides and $(1<4)-O$ disaccharide structures in order to study the influence of the oxygen atom of the $O$-glycosidic bond over the course of the sequential process.

In the case of four-atom $O$-glycosides, HAT at $\mathrm{C}$ - 1 was less efficient due to a more restricted conformation compared to its $C$-glycoside analogues. Also, because of possible electronic interactions between the nonbonding electron pairs of the $O$-glycoside fragment and the stannylated radical intermediates, a radical evolution by means of a $\beta$ fragmentation reaction was observed, giving mainly the more stable $\mathrm{C}-5$ radical, which was trapped by ATBT to yield a mixture of diastereomers. When a five-atom $O$ glycoside was submitted to the ATBT/AIBN protocol, the HAT reaction was expected to proceed preferentially at C5 as occurred with the TBTH/AIBN treatment, but because of the steric hindrance of the possible allylstannylated intermediate, in this case no abstraction took place, and the $O$ radical was prematurely trapped.

In the case of $(1<4)$ - $O$-disaccharides, the 6 - $O$-yl radical triggered a 1,8 -HAT reaction by exclusive abstraction of $5^{\prime}$ $\mathrm{H}$ on the pyranose and $4^{\prime}-\mathrm{H}$ on the furanose derivatives giving a C-radical which was mostly axially trapped by ATBT.

\section{Experimental Section}

General Methods: Melting points were determined with a hot-plate apparatus. Optical rotations were measured with a Perkin-Elmer Polarimeter PE-241 at the sodium line at ambient temperature in $\mathrm{CHCl}_{3}$. IR spectra were recorded with a Perkin-Elmer 1600/FTIR instrument in $\mathrm{CCl}_{4}$. NMR spectra were recorded with a Bruker AMX 400 spectrometer at $400 \mathrm{MHz}$ for ${ }^{1} \mathrm{H}$ and $100.6 \mathrm{MHz}$ for ${ }^{13} \mathrm{C}$ in $\mathrm{CDCl}_{3}$, unless otherwise stated, with TMS as internal standard. Mass spectra were recorded with a Waters LCT Premier XE spectrometer by using electrospray ionization (ESI+) or with a Micromass AutoSpec by using electron impact (EI) at $70 \mathrm{eV}$ or fast atom bombardment (FAB), as stated in each case. Elemental analyses were performed with a Leco TrueSpec Micro instrument. Merck silica gel $60 \mathrm{PF}(0.063-0.2 \mathrm{~mm})$ was used for column chromatography. Circular layers of $1 \mathrm{~mm}$ of Merck silica gel $60 \mathrm{PF}_{254}$ were used with a Chromatotron for centrifugally assisted chromatography. Commercially available reagents and solvents were of analytical grade or were purified by standard procedures prior to use. All reactions involving air- or moisture-sensitive materials were carried out under nitrogen. TLC analysis was conducted with a spray of $0.5 \%$ vanillin in $\mathrm{H}_{2} \mathrm{SO}_{4} / \mathrm{EtOH}$ (4:1) and heating until the development of a color.

General Procedure for the Reductive HAT/Radical Allylation: A solution of the corresponding phthalimide derivative $(1 \mathrm{mmol})$ in dry benzene $(10 \mathrm{~mL})$ was treated with allyltri- $n$-butyltin $(10 \mathrm{mmol})$ and AIBN $(0.01 \mathrm{mmol})$ and heated at reflux for a specific period of time (Tables 1,2, and 3). When the starting material was totally consumed, the reaction mixture was poured directly onto a silica gel chromatography column $[n$-hexanes/EtOAc + KF (10\% weight of the employed $\left.\mathrm{SiO}_{2}\right)$ ].

2-Hydroxyethyl 5-Allyl-5-deoxy-2,3,4,6-tetra-O-methyl-D-lyxohexonate (4): Compound 4 was obtained as a colourless oil in $48 \%$ yield as a diastereomeric mixture (1.1:1). ${ }^{1} \mathrm{H}$ NMR $(500 \mathrm{MHz}): \delta$ = 1.96-2.05 (m, $2 \mathrm{H}), 2.16-2.22(\mathrm{~m}, 3 \mathrm{H}), 2.27$ (m, $1 \mathrm{H}), 2.35$ (br. $\mathrm{s}, 2 \mathrm{H}), 2.29(\mathrm{~s}, 3 \mathrm{H}), 3.34(\mathrm{~s}, 3 \mathrm{H}), 3.34-3.38(\mathrm{~m}, 2 \mathrm{H}), 3.39(\mathrm{~s}, 3$ $\mathrm{H}), 3.41(\mathrm{~s}, 3 \mathrm{H}), 3.46(\mathrm{~s}, 3 \mathrm{H}), 3.48(\mathrm{~s}, 6 \mathrm{H}), 3.52(\mathrm{~s}, 3 \mathrm{H}), 3.57-$ $3.59(\mathrm{~m}, 2 \mathrm{H}), 3.63(\mathrm{dd}, J=7.2,3.4 \mathrm{~Hz}, 2 \mathrm{H}), 3.67(\mathrm{dd}, J=5.6$, $4.4 \mathrm{~Hz}, 1 \mathrm{H}), 3.77-3.85$ (m, $4 \mathrm{H}), 3.89$ (d, $J=3.4 \mathrm{~Hz}, 1 \mathrm{H}), 4.04$ $(\mathrm{d}, J=4.4 \mathrm{~Hz}, 1 \mathrm{H}), 4.13(\mathrm{ddd}, J=11.6,5.6,3.7 \mathrm{~Hz}, 1 \mathrm{H}), 4.27$ $(\mathrm{dd}, J=6.3,3.5 \mathrm{~Hz}, 1 \mathrm{H}), 4.30(\mathrm{dd}, J=6.0,3.2 \mathrm{~Hz}, 1 \mathrm{H}), 4.39$ $4.45(\mathrm{~m}, 2 \mathrm{H}), 5.01-5.09(\mathrm{~m}, 4 \mathrm{H}), 5.72-5.83(\mathrm{~m}, 2 \mathrm{H}) \mathrm{ppm} .{ }^{13} \mathrm{C}$ NMR (125.7 MHz): $\delta=30.4\left(\mathrm{CH}_{2}\right), 33.3\left(\mathrm{CH}_{2}\right), 40.2(\mathrm{CH}), 40.3$ (CH), $58.2\left(\mathrm{CH}_{3}\right), 58.4\left(\mathrm{CH}_{3}\right), 58.6\left(2 \times \mathrm{CH}_{3}\right), 60.4\left(\mathrm{CH}_{2}\right), 60.5$ $\left(\mathrm{CH}_{2}\right), 60.7\left(\mathrm{CH}_{3}\right), 60.8\left(\mathrm{CH}_{3}\right), 61.0\left(\mathrm{CH}_{3}\right), 61.4\left(\mathrm{CH}_{3}\right), 66.5\left(\mathrm{CH}_{2}\right)$, $66.8\left(\mathrm{CH}_{2}\right), 71.5\left(\mathrm{CH}_{2}\right), 72.4\left(\mathrm{CH}_{2}\right), 81.0(\mathrm{CH}), 81.4(\mathrm{CH}), 81.5$ $(\mathrm{CH}), 81.7(\mathrm{CH}), 84.3(\mathrm{CH}), 84.9(\mathrm{CH}), 116.4\left(\mathrm{CH}_{2}\right), 116.6\left(\mathrm{CH}_{2}\right)$, $136.7(2 \times \mathrm{CH}), 169.8(\mathrm{C}), 171.1(\mathrm{C}) \mathrm{ppm} . \mathrm{IR}\left(\mathrm{CCl}_{4}\right): \tilde{v}=3480$, $2929,1760,1450 \mathrm{~cm}^{-1}$. MS $\left(\mathrm{ESI}^{+}\right): \mathrm{m} / z(\%)=343(100)[\mathrm{M}+\mathrm{Na}]^{+}$. HRMS $\left(\mathrm{ESI}^{+}\right)$: calcd. for $\mathrm{C}_{15} \mathrm{H}_{28} \mathrm{NaO}_{7}[\mathrm{M}+\mathrm{Na}]^{+} 343.1733$; found 343.1730. $\mathrm{C}_{15} \mathrm{H}_{28} \mathrm{O}_{7}$ (320.38): calcd. C 56.23, $\mathrm{H}$ 8.81; found $\mathrm{C}$ 56.26, H 8.55 .

2-(Allyloxy)ethyl 2,3,4,6-Tetra- $\boldsymbol{O}$-acetyl- $\alpha$-D-mannopyranoside (6): Compound 6 was obtained as an amorphous solid in $33 \%$ yield from 5. $[\alpha]_{\mathrm{D}}=+20.6\left(c=0.17, \mathrm{CHCl}_{3}\right) .{ }^{1} \mathrm{H}$ NMR: $\delta=1.99(\mathrm{~s}, 3$ H), $2.03(\mathrm{~s}, 3 \mathrm{H}), 2.10(\mathrm{~s}, 3 \mathrm{H}), 2.15(\mathrm{~s}, 3 \mathrm{H}), 3.61-3.63(\mathrm{~m}, 2 \mathrm{H})$, $3.66(\mathrm{~m}, 1 \mathrm{H}), 3.82(\mathrm{ddd}, J=9.6,5.3,4.5 \mathrm{~Hz}, 1 \mathrm{H}), 4.02(\mathrm{ddd}, J=$ 5.6, 1.6, $1.6 \mathrm{~Hz}, 2 \mathrm{H}), 4.05-4.12(\mathrm{~m}, 2 \mathrm{H}), 4.28(\mathrm{dd}, J=12.2$, $5.0 \mathrm{~Hz}, 1 \mathrm{H}), 4.88(\mathrm{~d}, J=1.6 \mathrm{~Hz}, 1 \mathrm{H}), 5.19$ (dddd, $J=10.3,1.3$, 1.3, $1.3 \mathrm{~Hz}, 1 \mathrm{H}), 5.25-5.29(\mathrm{~m}, 2 \mathrm{H}), 5.30(\mathrm{dd}, J=3.4,1.8 \mathrm{~Hz}, 1$ H), 5.37 (dd, $J=9.8,3.4 \mathrm{~Hz}, 1 \mathrm{H}), 5.89$ (dddd, $J=17.2,10.3,5.6$, $5.6 \mathrm{~Hz}, 1 \mathrm{H}) \mathrm{ppm} .{ }^{13} \mathrm{C} \mathrm{NMR}: \delta=20.7\left(2 \times \mathrm{CH}_{3}\right), 20.7\left(\mathrm{CH}_{3}\right), 20.9$ $\left(\mathrm{CH}_{3}\right), 62.4\left(\mathrm{CH}_{2}\right), 66.2(\mathrm{CH}), 67.4(\mathrm{CH}), 68.4\left(\mathrm{CH}_{2}\right), 68.8(\mathrm{CH})$, $69.1(\mathrm{CH}), 69.6\left(\mathrm{CH}_{2}\right), 72.2\left(\mathrm{CH}_{2}\right), 97.7(\mathrm{CH}), 117.1\left(\mathrm{CH}_{2}\right), 134.5$ $(\mathrm{CH}), 169.7(\mathrm{C}), 169.9(\mathrm{C}), 170.0(\mathrm{C}), 170.7(\mathrm{C}) \mathrm{ppm}$. IR $\left(\mathrm{CCl}_{4}\right): \tilde{v}$ $=2960,1759,1644,1230 \mathrm{~cm}^{-1}$. MS $\left(\mathrm{ESI}^{+}\right): \mathrm{m} / \mathrm{z}(\%)=455(100)[\mathrm{M}$ $+\mathrm{Na}]^{+}$. HRMS $\left(\mathrm{ESI}^{+}\right)$: calcd. for $\mathrm{C}_{19} \mathrm{H}_{28} \mathrm{NaO}_{11}[\mathrm{M}+\mathrm{Na}]^{+}$ 455.1529; found 455.1526. $\mathrm{C}_{19} \mathrm{H}_{28} \mathrm{O}_{11}$ (432.42): calcd. C 52.77, $\mathrm{H}$ 6.53; found C 52.81, H 6.71 .

3-(Allyloxy)propyl $\quad 2,3,4,6$-Tetra- $O$-methyl- $\alpha$-D-mannopyranoside (11): Compound $\mathbf{1 1}$ was obtained as a colourless oil in $20 \%$ yield.

$[\alpha]_{\mathrm{D}}=+46.8\left(c=0.22, \mathrm{CHCl}_{3}\right) .{ }^{1} \mathrm{H} \mathrm{NMR}: \delta=1.86(\mathrm{dddd}, J=6.4$, 6.4, 6.4, $6.4 \mathrm{~Hz}, 2 \mathrm{H}), 3.37-3.59$ (m, $9 \mathrm{H}), 3.40$ (s, $3 \mathrm{H}), 3.47$ (s, 3 H), $3.50(\mathrm{~s}, 3 \mathrm{H}), 3.52(\mathrm{~s}, 3 \mathrm{H}), 3.79$ (ddd, $J=9.8,6.4,6.4 \mathrm{~Hz}, 1$ $\mathrm{H}), 3.96(\mathrm{ddd}, J=5.6,1.3,1.3 \mathrm{~Hz}, 2 \mathrm{H}), 4.87(\mathrm{~d}, J=1.9 \mathrm{~Hz}, 1 \mathrm{H})$, $5.17(\mathrm{~d}, J=10.3 \mathrm{~Hz}, 1 \mathrm{H}), 5.26(\mathrm{~d}, J=17.2 \mathrm{~Hz}, 1 \mathrm{H}), 5.91$ (dddd, $J=17.2,10.6,10.6,5.6 \mathrm{~Hz}, 1 \mathrm{H}) \mathrm{ppm} .{ }^{13} \mathrm{C} \mathrm{NMR}: \delta=29.8\left(\mathrm{CH}_{2}\right)$, $57.6\left(\mathrm{CH}_{3}\right), 58.8\left(\mathrm{CH}_{3}\right), 59.1\left(\mathrm{CH}_{3}\right), 60.6\left(\mathrm{CH}_{3}\right), 64.5\left(\mathrm{CH}_{2}\right), 67.0$ $\left(\mathrm{CH}_{2}\right), 71.2(\mathrm{CH}), 71.7\left(\mathrm{CH}_{2}\right), 71.8\left(\mathrm{CH}_{2}\right), 76.5(\mathrm{CH}), 77.2(\mathrm{CH})$, $81.2(\mathrm{CH}), 96.8(\mathrm{CH}), 116.7\left(\mathrm{CH}_{2}\right), 134.8(\mathrm{CH}) \mathrm{ppm}$. IR $\left(\mathrm{CCl}_{4}\right): \tilde{v}$ $=2930,1115 \mathrm{~cm}^{-1}$. MS $(70 \mathrm{eV}, \mathrm{EI}): \mathrm{m} / \mathrm{z}(\%)=219$ (2) $[\mathrm{M}-$ $\left.\mathrm{C}_{6} \mathrm{H}_{11} \mathrm{O}_{2}\right]^{+}, 159$ (56) $\left[\mathrm{M}-\mathrm{C}_{6} \mathrm{H}_{11} \mathrm{O}_{2}-\mathrm{C}_{2} \mathrm{H}_{4} \mathrm{O}\right]^{+}, 88$ (100). HRMS (EI): calcd. for $\mathrm{C}_{8} \mathrm{H}_{15} \mathrm{O}_{3}\left[\mathrm{M}-\mathrm{C}_{6} \mathrm{H}_{11} \mathrm{O}_{2}-\mathrm{C}_{2} \mathrm{H}_{4} \mathrm{O}\right]^{+} 159.1021$; found 159.1021. $\mathrm{C}_{16} \mathrm{H}_{30} \mathrm{O}_{7}$ (334.40): calcd. C 57.47, $\mathrm{H}$ 9.04; found $\mathrm{C}$ 57.47, H 9.09.

2-Hydroxyethyl 1,2,3-Trideoxy-5,6,7,9-tetra- $O$-methyl- $\beta$-D-gluconon-1-en-4-ulopyranoside (15): Compound 15 was obtained as a 
colourless oil in $13 \%$ yield from $\mathbf{1 4}$ and in $20 \%$ from $\mathbf{1 7}$ under the standard conditions. $[\alpha]_{\mathrm{D}}=+9.0\left(c=0.50, \mathrm{CHCl}_{3}\right) .{ }^{1} \mathrm{H}$ NMR $(500$ MHz): $\delta=1.97$ (br. s, $1 \mathrm{H}), 2.48(\mathrm{dd}, J=15.5,7.2 \mathrm{~Hz}, 1 \mathrm{H}$ ),

$2.61(\mathrm{dd}, J=15.5,5.3 \mathrm{~Hz}, 1 \mathrm{H}), 3.24(\mathrm{dd}, J=4.5,1.3 \mathrm{~Hz}, 2 \mathrm{H})$, $3.37(\mathrm{~s}, 3 \mathrm{H}), 3.38(\mathrm{~m}, 1 \mathrm{H}), 3.44(\mathrm{~m}, 1 \mathrm{H}), 3.51$ (s, $6 \mathrm{H}), 3.53$ (dd, $J=7.2,3.8 \mathrm{~Hz}, 2 \mathrm{H}), 3.58(\mathrm{~s}, 3 \mathrm{H}), 3.68(\mathrm{ddd}, J=4.1,4.1,0 \mathrm{~Hz}$, $2 \mathrm{H}), 3.75$ (ddd, $J=4.1,4.1,0 \mathrm{~Hz}, 2 \mathrm{H}), 5.09(\mathrm{~d}, J=10.1 \mathrm{~Hz}, 1$ $\mathrm{H}), 5.10(\mathrm{~d}, J=17.0 \mathrm{~Hz}, 1 \mathrm{H}), 5.84$ (dddd, $J=17.6,10.7,6.9$, $6.9 \mathrm{~Hz}, 1 \mathrm{H}) \mathrm{ppm}$; an NOE correlation was observed between 1'$\mathrm{H}_{2}$ with 3- and 5-H. ${ }^{13} \mathrm{C} \mathrm{NMR}(125.7 \mathrm{MHz}): \delta=35.7\left(\mathrm{CH}_{2}\right), 59.2$ $\left(\mathrm{CH}_{3}\right), 59.3\left(\mathrm{CH}_{3}\right), 59.9\left(\mathrm{CH}_{3}\right), 60.1\left(\mathrm{CH}_{3}\right), 63.1\left(\mathrm{CH}_{2}\right), 63.7\left(\mathrm{CH}_{2}\right)$, $71.4\left(\mathrm{CH}_{2}\right), 72.5(\mathrm{CH}), 79.2(\mathrm{CH}), 81.6(\mathrm{CH}), 85.6(\mathrm{CH}), 101.6(\mathrm{C})$, $118.0\left(\mathrm{CH}_{2}\right), 132.1(\mathrm{CH})$ ppm. IR $\left(\mathrm{CCl}_{4}\right): \tilde{v}=3502,2932$, $1709 \mathrm{~cm}^{-1}$. MS $(70 \mathrm{eV}, \mathrm{EI}): \mathrm{m} / z(\%)=279(95)\left[\mathrm{M}-\mathrm{C}_{3} \mathrm{H}_{5}\right]^{+}, 88$ (100). HRMS (EI): calcd. for $\mathrm{C}_{12} \mathrm{H}_{23} \mathrm{O}_{7}\left[\mathrm{M}-\mathrm{C}_{3} \mathrm{H}_{5}\right]^{+}$279.1444; found 279.1440. $\mathrm{C}_{15} \mathrm{H}_{28} \mathrm{O}_{7}$ (320.18): calcd. C 56.23, $\mathrm{H} \mathrm{8.81;} \mathrm{found}$ C 56.27, H 8.54.

2-Hydroxyethyl 5-Allyl-5-deoxy-2,3,4,6-tetra- $O$-methyl-D-xylohexonate (16): Compound $\mathbf{1 6}$ was obtained as a colourless oil in $40 \%$ yield from $\mathbf{1 4}$ and in $46 \%$ yield from $17 .{ }^{1} \mathrm{H}$ NMR: $\delta=1.32$ $(\mathrm{m}, 1 \mathrm{H}), 1.82(\mathrm{~m}, 1 \mathrm{H}), 1.94(\mathrm{~m}, 1 \mathrm{H}), 2.07-2.24(\mathrm{~m}, 2 \mathrm{H}), 2.32$ (ddd, $J=14.8,6.6,0 \mathrm{~Hz}, 1 \mathrm{H}), 2.50$ (br. s, $2 \mathrm{H}$ ), 3.27-3.38 (m, 2 H), $3.51(\mathrm{~s}, 3 \mathrm{H}), 3.40-3.52(\mathrm{~m}, 2 \mathrm{H}), 3.41(\mathrm{~s}, 3 \mathrm{H}), 3.44(\mathrm{~s}, 3 \mathrm{H})$, $3.45(\mathrm{~s}, 3 \mathrm{H}), 3.46(\mathrm{~s}, 3 \mathrm{H}), 3.47(\mathrm{~s}, 3 \mathrm{H}), 3.48(\mathrm{~s}, 3 \mathrm{H}), 3.51$ (s, 3 H), 3.53-3.59 (m, $2 \mathrm{H}), 3.61-3.69(\mathrm{~m}, 2 \mathrm{H}), 3.70-3.76(\mathrm{~m}, 2 \mathrm{H})$, $3.78-3.85(\mathrm{~m}, 2 \mathrm{H}), 3.96(\mathrm{~d}, J=4.2 \mathrm{~Hz}, 1 \mathrm{H}), 4.08(\mathrm{~d}, J=3.7 \mathrm{~Hz}$, $1 \mathrm{H}), 4.14(\mathrm{~m}, 1 \mathrm{H}), 4.27(\mathrm{~m}, 1 \mathrm{H}), 4.39(\mathrm{~m}, 1 \mathrm{H}), 4.49(\mathrm{~m}, 1 \mathrm{H})$, 5.00-5.12 (m, $4 \mathrm{H}), 5.71-5.83(\mathrm{~m}, 2 \mathrm{H}) \mathrm{ppm} .{ }^{13} \mathrm{C} \mathrm{NMR}: \delta=33.4$ $\left(\mathrm{CH}_{2}\right), 34.5\left(\mathrm{CH}_{2}\right), 39.8(\mathrm{CH}), 40.3(\mathrm{CH}), 58.5\left(\mathrm{CH}_{3}\right), 58.6\left(\mathrm{CH}_{3}\right)$, $58.9\left(2 \times \mathrm{CH}_{3}\right), 59.8\left(\mathrm{CH}_{3}\right), 59.9\left(\mathrm{CH}_{3}\right), 60.2\left(\mathrm{CH}_{3}\right), 60.6\left(\mathrm{CH}_{2}\right)$, $60.7\left(\mathrm{CH}_{3}\right), 60.8\left(\mathrm{CH}_{2}\right), 66.4\left(\mathrm{CH}_{2}\right), 66.6\left(\mathrm{CH}_{2}\right), 71.9\left(\mathrm{CH}_{2}\right), 72.4$ $\left(\mathrm{CH}_{2}\right), 80.2(\mathrm{CH}), 80.3(\mathrm{CH}), 81.0(\mathrm{CH}), 81.5(\mathrm{CH}), 82.6(\mathrm{CH})$, $83.8(\mathrm{CH}), 116.3\left(\mathrm{CH}_{2}\right), 116.4\left(\mathrm{CH}_{2}\right), 135.0(\mathrm{CH}), 136.9(\mathrm{CH})$, 170.8 (C), 171.0 (C) ppm. IR $\left(\mathrm{CCl}_{4}\right): \tilde{v}=3488,2929,1752$, $1448 \mathrm{~cm}^{-1}$. MS $\left(\mathrm{ESI}^{+}\right): \mathrm{m} / z(\%)=343(100)[\mathrm{M}+\mathrm{Na}]^{+}$. HRMS $\left(\mathrm{ESI}^{+}\right)$: calcd. for $\mathrm{C}_{15} \mathrm{H}_{28} \mathrm{NaO}_{7}[\mathrm{M}+\mathrm{Na}]^{+} 343.1733$; found 343.1733. $\mathrm{C}_{15} \mathrm{H}_{28} \mathrm{O}_{7}$ (320.38): calcd. C 56.23, $\mathrm{H}$ 8.81; found $\mathrm{C}$ $56.24, \mathrm{H} 8.72$.

Methyl 2,3-Di- $O$-methyl-4- $O$-(6,7,8-trideoxy-5-methyl-2,3,4-tri- $O$ methyl- $\beta$-D-gulo-oct-7-enopyranosyl)- $\alpha$-D-galactopyranoside (19): Compound 19 was obtained as a colourless oil in $51 \%$ yield from

18. $[\alpha]_{\mathrm{D}}=+29.1\left(c=0.57, \mathrm{CHCl}_{3}\right) \cdot{ }^{1} \mathrm{H}$ NMR $(500 \mathrm{MHz}): \delta=1.27$ $(\mathrm{s}, 3 \mathrm{H}), 2.37(\mathrm{dd}, J=11.6,5.6 \mathrm{~Hz}, 1 \mathrm{H}), 2.69(\mathrm{dd}, J=11.6,6.0 \mathrm{~Hz}$, $1 \mathrm{H}), 3.21(\mathrm{~d}, J=6.0 \mathrm{~Hz}, 1 \mathrm{H}), 3.40(\mathrm{~s}, 3 \mathrm{H}), 3.47(\mathrm{~s}, 3 \mathrm{H}), 3.49(\mathrm{~s}$, $3 \mathrm{H}), 3.50(\mathrm{~s}, 3 \mathrm{H}), 3.51(\mathrm{~s}, 3 \mathrm{H}), 3.54(\mathrm{dd}, J=5.6,3.6 \mathrm{~Hz}, 1 \mathrm{H})$, 3.57 (s, $3 \mathrm{H}), 3.57-3.59(\mathrm{~m}, 2 \mathrm{H}), 3.63(\mathrm{dd}, J=9.3,4.3 \mathrm{~Hz}, 1 \mathrm{H})$, $3.69(\mathrm{dd}, J=5.8,3.5 \mathrm{~Hz}, 1 \mathrm{H}), 3.74-3.82(\mathrm{~m}, 2 \mathrm{H}), 4.20(\mathrm{~m}, 1 \mathrm{H})$, $4.83(\mathrm{~d}, J=2.4 \mathrm{~Hz}, 1 \mathrm{H}), 5.09(\mathrm{~d}, J=5.5 \mathrm{~Hz}, 1 \mathrm{H}), 5.12(\mathrm{~d}, J=$ $13.6 \mathrm{~Hz}, 1 \mathrm{H}), 5.15(\mathrm{~d}, J=19.2 \mathrm{~Hz}, 1 \mathrm{H}), 5.85(\mathrm{~m}, 1 \mathrm{H}) \mathrm{ppm}$; the signal for $1 \mathrm{H}$ from an $\mathrm{OH}$ group is missing, an NOE correlation was observed between 4 '- $\mathrm{H}$ and $6{ }^{\prime}-\mathrm{H}_{3} .{ }^{13} \mathrm{C}$ NMR $(125.7 \mathrm{MHz}): \delta$ $=22.3\left(\mathrm{CH}_{3}\right), 41.6\left(\mathrm{CH}_{2}\right), 55.6\left(\mathrm{CH}_{3}\right), 58.8\left(\mathrm{CH}_{3}\right), 59.1\left(\mathrm{CH}_{3}\right), 59.5$ $\left(\mathrm{CH}_{3}\right), 59.7\left(\mathrm{CH}_{3}\right), 60.4\left(\mathrm{CH}_{3}\right), 60.9\left(\mathrm{CH}_{2}\right), 69.9(\mathrm{CH}), 73.1(\mathrm{CH})$, $78.3(\mathrm{CH}), 78.7(\mathrm{CH}), 79.0(\mathrm{CH}), 79.4(\mathrm{C}), 80.2(2 \times \mathrm{CH}), 98.5$ $(\mathrm{CH}), 99.2(\mathrm{CH}), 118.6\left(\mathrm{CH}_{2}\right), 133.4(\mathrm{CH}) \mathrm{ppm}$. IR $\left(\mathrm{CCl}_{4}\right): \tilde{v}=$ 3489, 2931, 1741, $1434 \mathrm{~cm}^{-1}$. MS (70 eV, EI): $\mathrm{m} / z(\%)=409(29)$ $\left[\mathrm{M}-\mathrm{C}_{3} \mathrm{H}_{5}\right]^{+}, 88$ (100). HRMS (EI): calcd. for $\mathrm{C}_{18} \mathrm{H}_{33} \mathrm{O}_{10}[\mathrm{M}-$ $\left.\mathrm{C}_{3} \mathrm{H}_{5}\right]^{+}$409.2074; found 409.2072. $\mathrm{C}_{21} \mathrm{H}_{38} \mathrm{O}_{10}$ (450.25): calcd. $\mathrm{C}$ 55.99, H 8.50; found C 55.96, H 8.37.

Methyl (5'R)-5',6-Anhydro-4- $O$-(4,6-dideoxy-2',3'-di- $O$-methyl- $\beta$-Derythro-hexose-5'-ulopyranosyl)-2,3-di- $O$-methyl- $\alpha$-D-galactopyranoside (20): Compound 20 was obtained as a colourless oil in
$36 \%$ yield from 18. $[\alpha]_{\mathrm{D}}=+103.3\left(c=0.12, \mathrm{CHCl}_{3}\right)$. ${ }^{1} \mathrm{H}$ NMR: $\delta$ $=1.38(\mathrm{~s}, 3 \mathrm{H}), 1.94(\mathrm{dd}, J=12.5,12.5 \mathrm{~Hz}, 1 \mathrm{H}), 2.10(\mathrm{dd}, J=$ 12.7, $4.8 \mathrm{~Hz}, 1 \mathrm{H}), 3.40$ (s, $3 \mathrm{H}), 3.42(\mathrm{~s}, 3 \mathrm{H}), 3.48-3.55(\mathrm{~m}, 2 \mathrm{H})$, $3.49(\mathrm{~s}, 3 \mathrm{H}), 3.52(\mathrm{~s}, 3 \mathrm{H}), 3.54(\mathrm{~s}, 3 \mathrm{H}), 3.62(\mathrm{dd}, J=10.0,3.7 \mathrm{~Hz}$, $1 \mathrm{H}), 3.75(\mathrm{~m}, 1 \mathrm{H}), 3.79(\mathrm{dd}, J=13.5,2.4 \mathrm{~Hz}, 1 \mathrm{H}), 3.97(\mathrm{ddd}, J$ $=11.9,4.8,2.9 \mathrm{~Hz}, 1 \mathrm{H}), 4.10(\mathrm{dd}, J=13.5,1.3 \mathrm{~Hz}, 1 \mathrm{H}), 4.18(\mathrm{dd}$, $J=3.2,0 \mathrm{~Hz}, 1 \mathrm{H}), 4.97(\mathrm{~d}, J=3.7 \mathrm{~Hz}, 1 \mathrm{H}), 5.02(\mathrm{~d}, J=1.8 \mathrm{~Hz}$, $1 \mathrm{H})$ ppm. ${ }^{13} \mathrm{C}$ NMR: $\delta=22.2\left(\mathrm{CH}_{3}\right), 36.0\left(\mathrm{CH}_{2}\right), 55.7\left(\mathrm{CH}_{3}\right), 56.4$ $\left(\mathrm{CH}_{3}\right), 58.4\left(\mathrm{CH}_{3}\right), 59.0\left(\mathrm{CH}_{3}\right), 59.6\left(\mathrm{CH}_{3}\right), 63.7\left(\mathrm{CH}_{2}\right), 67.8(\mathrm{CH})$, $72.5(\mathrm{CH}), 73.9(\mathrm{CH}), 74.8(\mathrm{CH}), 78.9(\mathrm{CH}), 82.4(\mathrm{CH}), 98.4(\mathrm{CH})$, $99.1(\mathrm{CH}), 100.8(\mathrm{C}) \mathrm{ppm}$. IR $\left(\mathrm{CCl}_{4}\right): \tilde{v}=2930,1737,1385 \mathrm{~cm}^{-1}$. MS (70 eV, EI): $\mathrm{m} / z(\%)=378(18)[\mathrm{M}]^{+}, 88$ (100). HRMS (EI): calcd. for $\mathrm{C}_{17} \mathrm{H}_{30} \mathrm{O}_{9}[\mathrm{M}]+378.1890$; found 378.1886. $\mathrm{C}_{17} \mathrm{H}_{30} \mathrm{O}_{9}$ (378.41): calcd. C 53.96, H 7.99; found C 54.07, H 7.97.

Methyl 2,3-Di- $O$-methyl-4- $O$-(6,7,8-trideoxy-5-methyl-2,3,4-tri- $O$ acetyl- $\beta$-D-gulo-oct-7-enopyranosyl)- $\alpha$-D-galactopyranoside (22): Compound 22 was obtained as a colourless oil in $42 \%$ yield from 21 under the standard conditions. $[\alpha]_{\mathrm{D}}=+35.4\left(c=1.00, \mathrm{CHCl}_{3}\right)$. ${ }^{1} \mathrm{H}$ NMR: $\delta=1.26(\mathrm{~s}, 3 \mathrm{H}), 2.07(\mathrm{~s}, 3 \mathrm{H}), 2.10(\mathrm{~s}, 3 \mathrm{H}), 2.13(\mathrm{~s}, 3$ H), $2.43(\mathrm{dd}, J=14.6,7.2 \mathrm{~Hz}, 1 \mathrm{H}), 2.70(\mathrm{dd}, J=14.3,7.1 \mathrm{~Hz}, 1$ H), $3.40(\mathrm{~s}, 3 \mathrm{H}), 3.48(\mathrm{~s}, 3 \mathrm{H}), 3.51(\mathrm{~s}, 3 \mathrm{H}), 3.52(\mathrm{dd}, J=4.8$, $3.4 \mathrm{~Hz}, 1 \mathrm{H}), 3.54(\mathrm{dd}, J=5.0,2.6 \mathrm{~Hz}, 2 \mathrm{H}), 3.68-3.79(\mathrm{~m}, 3 \mathrm{H})$, $4.12(\mathrm{dd}, J=5.0,2.6 \mathrm{~Hz}, 1 \mathrm{H}), 4.82(\mathrm{~d}, J=3.2 \mathrm{~Hz}, 1 \mathrm{H}), 5.03(\mathrm{~d}$, $J=5.6 \mathrm{~Hz}, 1 \mathrm{H}), 5.08(\mathrm{~d}, J=6.4 \mathrm{~Hz}, 1 \mathrm{H}), 5.16(\mathrm{dd}, J=16.9$, $1.8 \mathrm{~Hz}, 1 \mathrm{H}), 5.21(\mathrm{dd}, J=10.3,1.8 \mathrm{~Hz}, 1 \mathrm{H}), 5.28(\mathrm{dd}, J=6.4$, $3.6 \mathrm{~Hz}, 1 \mathrm{H}), 5.38(\mathrm{dd}, J=5.6,3.7 \mathrm{~Hz}, 1 \mathrm{H}), 5.84(\mathrm{dddd}, J=17.2$, 10.0, 7.1, 7.1 Hz, $1 \mathrm{H}) \mathrm{ppm}$; an NOE correlation was observed between $4^{\prime}-\mathrm{H}$ and $6^{\prime}-\mathrm{H}_{3}$, and between $7{ }^{\prime}-\mathrm{H}_{2}$ and $2^{\prime}-\mathrm{H}$ or $3^{\prime}-\mathrm{H} .{ }^{13} \mathrm{C}$ NMR: $\delta=14.6\left(\mathrm{CH}_{3}\right), 21.1\left(2 \times \mathrm{CH}_{3}\right), 23.0\left(\mathrm{CH}_{3}\right), 41.5\left(\mathrm{CH}_{2}\right)$, $55.7\left(\mathrm{CH}_{3}\right), 59.0\left(\mathrm{CH}_{3}\right), 59.6\left(\mathrm{CH}_{3}\right), 61.2\left(\mathrm{CH}_{2}\right), 68.8(\mathrm{CH}), 68.9$ $(\mathrm{CH}), 69.8(\mathrm{CH}), 71.0(\mathrm{CH}), 74.9(\mathrm{CH}), 78.5(\mathrm{C}), 78.5(\mathrm{CH}), 79.8$ $(\mathrm{CH}), 97.9(\mathrm{CH}), 98.4(\mathrm{CH}), 119.9\left(\mathrm{CH}_{2}\right), 132.4(\mathrm{CH}), 169.5(\mathrm{C})$, $169.9(2 \times \mathrm{C}) \mathrm{ppm}$. IR $\left(\mathrm{CCl}_{4}\right): \tilde{v}=3511,2933,1755,1223 \mathrm{~cm}^{-1}$. MS (70 eV, EI): $\mathrm{m} / z(\%)=475(13)[\mathrm{M}-\mathrm{OAc}]^{+}, 429(100)\left[\mathrm{M}-\mathrm{C}_{5}\right.$ $\left.\mathrm{H}_{13} \mathrm{O}_{2}\right]^{+}$. $\operatorname{HRMS}$ (EI): calcd. for $\mathrm{C}_{22} \mathrm{H}_{35} \mathrm{O}_{11}[\mathrm{M}-\mathrm{OAc}]^{+}$ 475.2179; found 475.2160. $\mathrm{C}_{24} \mathrm{H}_{38} \mathrm{O}_{13}$ (534.55): calcd. C 53.93, $\mathrm{H}$ 7.17; found C 53.80, H 7.12.

Methyl 6- $O$-Allyl-2,3-di- $O$-methyl-4- $O$-(2,3,4-tri- $O$-acetyl-6-deoxy$\alpha$-L-mannopyranosyl)- $\alpha$-D-galactopyranoside (23): Compound 23 was obtained as a colourless oil in $11 \%$ yield from 21. $[\alpha]_{\mathrm{D}}=+18.2$ $\left(c=0.50, \mathrm{CHCl}_{3}\right) .{ }^{1} \mathrm{H}$ NMR: $\delta=1.35(\mathrm{~d}, J=7.4 \mathrm{~Hz}, 3 \mathrm{H}), 1.99$ $(\mathrm{s}, 3 \mathrm{H}), 2.05(\mathrm{~s}, 3 \mathrm{H}), 2.14(\mathrm{~s}, 3 \mathrm{H}), 3.42(\mathrm{~s}, 3 \mathrm{H}), 3.47(\mathrm{~s}, 3 \mathrm{H})$, $3.53(\mathrm{~s}, 3 \mathrm{H}), 3.53(\mathrm{~m}, 1 \mathrm{H}), 3.56(\mathrm{dd}, J=6.3,2.4 \mathrm{~Hz}, 1 \mathrm{H}), 3.59$ $(\mathrm{dd}, J=9.2,6.9 \mathrm{~Hz}, 1 \mathrm{H}), 3.66(\mathrm{dd}, J=9.8,3.4 \mathrm{~Hz}, 1 \mathrm{H}), 3.91$ (ddd, $J=6.4,6.4,0 \mathrm{~Hz}, 1 \mathrm{H}), 3.97(\mathrm{~m}, 1 \mathrm{H}), 4.03(\mathrm{~d}, J=5.6 \mathrm{~Hz}$, $2 \mathrm{H}), 4.16(\mathrm{dd}, J=2.4,0 \mathrm{~Hz}, 1 \mathrm{H}), 4.87(\mathrm{~d}, J=3.4 \mathrm{~Hz}, 1 \mathrm{H}), 5.07$ $(\mathrm{dd}, J=10.1,10.1 \mathrm{~Hz}, 1 \mathrm{H}), 5.15(\mathrm{~d}, J=1.9 \mathrm{~Hz}, 1 \mathrm{H}), 5.19(\mathrm{dd}, J$ $=10.3,1.1 \mathrm{~Hz}, 1 \mathrm{H}), 5.28(\mathrm{dd}, J=16.7,1.1 \mathrm{~Hz}, 1 \mathrm{H}), 5.32(\mathrm{dd}, J$ $=9.8,2.9 \mathrm{~Hz}, 1 \mathrm{H}), 5.47(\mathrm{dd}, J=3.2,3.2 \mathrm{~Hz}, 1 \mathrm{H}), 5.91(\mathrm{~m}, 1 \mathrm{H})$ ppm. ${ }^{13} \mathrm{C}$ NMR: $\delta=17.9\left(\mathrm{CH}_{3}\right), 21.1\left(\mathrm{CH}_{3}\right), 21.2\left(\mathrm{CH}_{3}\right), 21.3$ $\left(\mathrm{CH}_{3}\right), 55.7\left(\mathrm{CH}_{3}\right), 58.9\left(\mathrm{CH}_{3}\right), 59.6\left(\mathrm{CH}_{3}\right), 67.3(\mathrm{CH}), 68.9(\mathrm{CH})$, $69.2\left(\mathrm{CH}_{2}\right), 69.5\left(\mathrm{CH}_{2}\right), 70.3(\mathrm{CH}), 71.5(\mathrm{CH}), 72.8(\mathrm{CH}), \quad 73.8$ $(\mathrm{CH}), 78.2(\mathrm{CH}), 80.7(\mathrm{CH}), 98.5(\mathrm{CH}), 99.2(\mathrm{CH}), 117.6\left(\mathrm{CH}_{2}\right)$, $134.8(\mathrm{CH}), 170.3(3 \times \mathrm{C}) \mathrm{ppm}$. IR $\left(\mathrm{CCl}_{4}\right): \tilde{v}=2932,1752$, $1223 \mathrm{~cm}^{-1}$. MS $(70 \mathrm{eV}, \mathrm{EI}): \mathrm{m} / z(\%)=434(8)\left[\mathrm{M}-\mathrm{C}_{3} \mathrm{H}_{5}-\mathrm{OAc}\right]^{+}$, 88 (100). HRMS (EI): calcd. for $\mathrm{C}_{19} \mathrm{H}_{30} \mathrm{O}_{11}\left[\mathrm{M}-\mathrm{C}_{3} \mathrm{H}_{5}-\mathrm{OAc}\right]^{+}$ 434.1788; found 434.1804. $\mathrm{C}_{24} \mathrm{H}_{38} \mathrm{O}_{13}$ (534.55): calcd. C 53.93, $\mathrm{H}$ 7.17; found C 54.18, H 6.98 .

Methyl (6S)-7,8,9-Trideoxy-2,3-di- $O$-methyl-4- $O$-(2,3,4-tri- $O$ acetyl-6-deoxy- $\alpha$-L-mannopyranosyl)-L-glycero- $\alpha$-D-galacto-non-8enopyranoside (24): Compound $\mathbf{2 4}$ was obtained as a colourless oil in $10 \%$ yield from 21. $[\alpha]_{\mathrm{D}}=+69.0\left(c=0.51, \mathrm{CHCl}_{3}\right) .{ }^{1} \mathrm{H}$ NMR: 
$\delta=1.33(\mathrm{~d}, J=6.4 \mathrm{~Hz}, 3 \mathrm{H}), 2.00(\mathrm{~s}, 3 \mathrm{H}), 2.06(\mathrm{~s}, 3 \mathrm{H}), 2.15(\mathrm{~s}$, $3 \mathrm{H}), 2.21(\mathrm{~m}, 1 \mathrm{H}), 2.56$ (br. s, $1 \mathrm{H}), 2.58(\mathrm{~m}, 1 \mathrm{H}), 3.41(\mathrm{~s}, 3 \mathrm{H})$, $3.47(\mathrm{~s}, 3 \mathrm{H}), 3.48(\mathrm{dd}, J=6.9,1.6 \mathrm{~Hz}, 1 \mathrm{H}), 3.52(\mathrm{dd}, J=9.3$, $2.9 \mathrm{~Hz}, 1 \mathrm{H}), 3.53(\mathrm{~s}, 3 \mathrm{H}), 3.62(\mathrm{dd}, J=10.1,3.4 \mathrm{~Hz}, 1 \mathrm{H}), 3.74$ $(\mathrm{m}, 1 \mathrm{H}), 4.10(\mathrm{dddd}, J=9.6,6.4,6.4,6.4 \mathrm{~Hz}, 1 \mathrm{H}), 4.30(\mathrm{dd}, J=$ $6.7,1.1 \mathrm{~Hz}, 1 \mathrm{H}), 4.84(\mathrm{~d}, J=3.7 \mathrm{~Hz}, 1 \mathrm{H}), 5.02(\mathrm{~d}, J=2.1 \mathrm{~Hz}, 1$ $\mathrm{H}), 5.08(\mathrm{dd}, J=9.8,9.8 \mathrm{~Hz}, 1 \mathrm{H}), 5.15-5.21(\mathrm{~m}, 2 \mathrm{H}), 5.35(\mathrm{dd}$, $J=9.8,3.5 \mathrm{~Hz}, 1 \mathrm{H}), 5.49(\mathrm{dd}, J=3.2,2.1 \mathrm{~Hz}, 1 \mathrm{H}), 5.91(\mathrm{~m}, 1$ H) ppm. ${ }^{13} \mathrm{C}$ NMR: $\delta=17.5\left(\mathrm{CH}_{3}\right), 20.8\left(2 \times \mathrm{CH}_{3}\right), 21.0\left(\mathrm{CH}_{3}\right)$, $38.0\left(\mathrm{CH}_{2}\right), 55.5\left(\mathrm{CH}_{3}\right), 58.6\left(\mathrm{CH}_{3}\right), 59.3\left(\mathrm{CH}_{3}\right), 67.9(\mathrm{CH}), 68.7$ $(\mathrm{CH}), 69.0(\mathrm{CH}), 69.9(\mathrm{CH}), 71.0(\mathrm{CH}), 71.9(\mathrm{CH}), 76.2(\mathrm{CH}), 77.9$ $(\mathrm{CH}), 79.5(\mathrm{CH}), 98.2(\mathrm{CH}), 100.5(\mathrm{CH}), 118.2\left(\mathrm{CH}_{2}\right), 134.5(\mathrm{CH})$, $170.3(3 \times \mathrm{C})$ ppm. IR $\left(\mathrm{CCl}_{4}\right): \tilde{v}=3527,2933,1753,1223 \mathrm{~cm}^{-1}$. MS $\left(\mathrm{FAB}^{+}\right): m / z(\%)=557(2)\left[\mathrm{M}+\mathrm{Na}^{+}, 273\right.$ (100). HRMS $\left(\mathrm{FAB}^{+}\right)$: calcd. for $\mathrm{C}_{24} \mathrm{H}_{38} \mathrm{O}_{13}[\mathrm{M}+\mathrm{Na}]^{+}$557.2210; found 557.2218. $\mathrm{C}_{24} \mathrm{H}_{38} \mathrm{O}_{13}$ (534.55): calcd. C 53.93, H 7.17; found C 54.29, H 7.18.

Methyl 2,3-Di- $O$-methyl-4- $O$-(6,7,8-trideoxy-5-methyl-2,3,4-tri- $O$ -

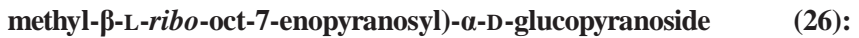
Compound 26 was obtained as a colourless oil from 25 in $53 \%$ yield as a mixture of diastereomers $\left(5^{\prime} S / 5^{\prime} R 3: 1\right) .{ }^{1} \mathrm{H}$ NMR: $\delta=$ 1.25 (s, $3 \mathrm{H}), 1.37$ (s, $3 \mathrm{H}), 1.65$ (br. s, $2 \mathrm{H}), 2.20-2.31(\mathrm{~m}, 2 \mathrm{H})$, $2.86(\mathrm{dd}, J=7.6,2.3 \mathrm{~Hz}, 1 \mathrm{H}), 2.95(\mathrm{dd}, J=6.8,3.5 \mathrm{~Hz}, 1 \mathrm{H})$, $3.12-3.26(\mathrm{~m}, 6 \mathrm{H}), 3.40-3.66(\mathrm{~m}, 8 \mathrm{H}), 3.38$ (s, $3 \mathrm{H}), 3.39$ (s, $3 \mathrm{H})$, $3.40(\mathrm{~s}, 3 \mathrm{H}), 3.44(\mathrm{~s}, 3 \mathrm{H}), 3.51(\mathrm{~s}, 3 \mathrm{H}), 3.52(\mathrm{~s}, 6 \mathrm{H}), 3.54(\mathrm{~s}, 3$ $\mathrm{H}), 3.57(\mathrm{~s}, 3 \mathrm{H}), 3.58(\mathrm{~s}, 3 \mathrm{H}), 3.63(\mathrm{~s}, 3 \mathrm{H}), 3.64(\mathrm{~s}, 3 \mathrm{H}), 3.90$ $3.95(\mathrm{~m}, 2 \mathrm{H}), 4.03(\mathrm{dd}, J=2.8,2.8 \mathrm{~Hz}, 2 \mathrm{H}), 4.81(\mathrm{~d}, J=3.3 \mathrm{~Hz}$, $2 \mathrm{H}), 5.07-5.23(\mathrm{~m}, 6 \mathrm{H}), 5.82-5.92(\mathrm{~m}, 2 \mathrm{H}) \mathrm{ppm}$; an NOE correlation was observed between $6^{\prime}-\mathrm{H}_{3}$ and $3^{\prime}-\mathrm{OMe}$ for the major $\left(5^{\prime} S\right)$ isomer. ${ }^{13} \mathrm{C} \mathrm{NMR:} \delta=19.7\left(\mathrm{CH}_{3}\right), 25.5\left(\mathrm{CH}_{3}\right), 35.6\left(\mathrm{CH}_{2}\right), 45.6$ $\left(\mathrm{CH}_{2}\right), 55.1\left(2 \times \mathrm{CH}_{3}\right), 56.9\left(\mathrm{CH}_{3}\right), 57.8\left(\mathrm{CH}_{3}\right), 59.0\left(3 \times \mathrm{CH}_{3}\right)$, $59.2\left(\mathrm{CH}_{3}\right), 60.9\left(\mathrm{CH}_{3}\right), 61.1\left(3 \times \mathrm{CH}_{3}\right), 62.0\left(2 \times \mathrm{CH}_{2}\right), 70.0(\mathrm{CH})$, $70.2(\mathrm{CH}), 75.3(\mathrm{CH}), 75.5(\mathrm{CH}), 75.7(2 \times \mathrm{CH}), 77.9(\mathrm{C}), 78.2(\mathrm{C})$, $80.8(\mathrm{CH}), 81.3(\mathrm{CH}), 82.1(2 \times \mathrm{CH}), 83.4(\mathrm{CH}), 83.6(\mathrm{CH}), 85.3$ $(2 \times \mathrm{CH}), 97.1(2 \times \mathrm{CH}), 97.6(\mathrm{CH}), 97.8(\mathrm{CH}), 118.5\left(\mathrm{CH}_{2}\right), 119.0$ $\left(\mathrm{CH}_{2}\right), 133.3(\mathrm{CH}), 133.4(\mathrm{CH}) \mathrm{ppm}$. IR $\left(\mathrm{CCl}_{4}\right): \tilde{v}=3504,2931$, $1445,1105 \mathrm{~cm}^{-1}$. MS (70 eV, EI): $m / z(\%)=409(19)\left[\mathrm{M}-\mathrm{C}_{3} \mathrm{H}_{5}\right]^{+}$, 88 (100). HRMS (EI): calcd. for $\mathrm{C}_{18} \mathrm{H}_{33} \mathrm{O}_{10}\left[\mathrm{M}-\mathrm{C}_{3} \mathrm{H}_{5}\right]+409.2074$; found 409.2075. $\mathrm{C}_{21} \mathrm{H}_{38} \mathrm{O}_{10}$ (450.52): calcd. C 55.99, $\mathrm{H}$ 8.50; found C 55.96, H 8.39.

Methyl 2,3-Di- $O$-acetyl-6,7,8-trideoxy-4- $O$-(2,3,4,6-tetra- $O$-acetyl$\alpha$-D-glucopyranosyl)- $\beta$-D-xylo-oct-7-enopyranoside (28): Compound $\mathbf{2 8}$ was obtained as an amorphous solid in $68 \%$ yield from $\mathbf{2 7}$ as a mixture of diastereomers $(5 R / 5 S, 2.5: 1) .{ }^{1} \mathrm{H}$ NMR: $\delta=2.00(\mathrm{~s}, 6$ $\mathrm{H}), 2.01(\mathrm{~s}, 12 \mathrm{H}), 2.04(\mathrm{~s}, 12 \mathrm{H}), 2.10(\mathrm{~s}, 6 \mathrm{H}), 2.43$ (ddd, $J=14.4$, 7.3, 7.3 Hz, $2 \mathrm{H}$ ), 2.55 (ddd, $J=13.9,7.1,7.1 \mathrm{~Hz}, 2 \mathrm{H}$ ), 3.37 (ddd, $J=8.4,0,0 \mathrm{~Hz}, 1 \mathrm{H}), 3.48(\mathrm{~s}, 3 \mathrm{H}), 3.49(\mathrm{~s}, 3 \mathrm{H}), 3.54(\mathrm{~m}, 1 \mathrm{H})$, $3.70(\mathrm{dd}, J=11.6,1.8 \mathrm{~Hz}, 1 \mathrm{H}), 3.81(\mathrm{dd}, J=11.6,3.5 \mathrm{~Hz}, 1 \mathrm{H})$, $3.90(\mathrm{dd}, J=15.4,7.3 \mathrm{~Hz}, 2 \mathrm{H}), 4.01-4.12(\mathrm{~m}, 2 \mathrm{H}), 4.21(\mathrm{dd}, J=$ 9.4, $9.4 \mathrm{~Hz}, 1 \mathrm{H}), 4.30(\mathrm{dd}, J=12.4,4.0 \mathrm{~Hz}, 1 \mathrm{H}), 4.38(\mathrm{~d}, J=$ $7.8 \mathrm{~Hz}, 1 \mathrm{H}), 4.39(\mathrm{~d}, J=7.8 \mathrm{~Hz}, 1 \mathrm{H}), 4.77(\mathrm{dd}, J=9.4,7.8 \mathrm{~Hz}$, $2 \mathrm{H}), 4.83(\mathrm{dd}, J=10.6,4.0 \mathrm{~Hz}, 2 \mathrm{H}), 5.02(\mathrm{dd}, J=9.8,9.8 \mathrm{~Hz}, 1$ H), $5.04(\mathrm{dd}, J=9.6,9.6 \mathrm{~Hz}, 1 \mathrm{H}), 5.15(\mathrm{~d}, J=11.1 \mathrm{~Hz}, 2 \mathrm{H}), 5.16$ $(\mathrm{d}, J=16.4 \mathrm{~Hz}, 2 \mathrm{H}), 5.26(\mathrm{dd}, J=9.4,9.4 \mathrm{~Hz}, 2 \mathrm{H}), 5.36(\mathrm{dd}, J$ $=10.1,10.1 \mathrm{~Hz}, 2 \mathrm{H}), 5.42(\mathrm{~d}, J=4.0 \mathrm{~Hz}, 1 \mathrm{H}), 5.47(\mathrm{~d}, J=4.1 \mathrm{~Hz}$, $1 \mathrm{H}), 5.79-5.94(\mathrm{~m}, 2 \mathrm{H}) \mathrm{ppm}$; an NOE correlation was observed between $5-\mathrm{H}$ and $1-\mathrm{H}$ and $3-\mathrm{H}$ for the major $(5 R)$ isomer. ${ }^{13} \mathrm{C}$ NMR: $\delta=20.5\left(6 \times \mathrm{CH}_{3}\right), 20.6\left(2 \times \mathrm{CH}_{3}\right), 20.7\left(2 \times \mathrm{CH}_{3}\right), 20.9$ $\left(2 \times \mathrm{CH}_{3}\right), 38.3\left(2 \times \mathrm{CH}_{2}\right), 56.7\left(\mathrm{CH}_{3}\right), 57.1\left(\mathrm{CH}_{3}\right), 61.6\left(\mathrm{CH}_{2}\right)$, $61.9\left(\mathrm{CH}_{2}\right), 67.8(\mathrm{CH}), 68.0(\mathrm{CH}), 68.2(\mathrm{CH}), 68.3(\mathrm{CH}), 69.5(\mathrm{CH})$, 69.6 (CH), 70.2 (CH), 70.3 (CH), 71.1 (CH), 71.4 (CH), 72.1 (CH), $72.4(\mathrm{CH}), 74.4(\mathrm{CH}), 74.7(\mathrm{CH}), 75.5(\mathrm{CH}), 75.6(\mathrm{CH}), 94.9(\mathrm{CH})$, $95.1(\mathrm{CH}), 101.3(\mathrm{CH}), 101.6(\mathrm{CH}), 117.4\left(\mathrm{CH}_{2}\right), 118.2\left(\mathrm{CH}_{2}\right)$, $133.9(\mathrm{CH}), 134.3(\mathrm{CH}), 169.4(2 \times \mathrm{C}), 169.6(2 \times \mathrm{C}), 170.0(2 \times$
C), $170.3(2 \times \mathrm{C}), 170.5(2 \times \mathrm{C}), 170.7(2 \times \mathrm{C}) \mathrm{ppm}$. IR $\left(\mathrm{CCl}_{4}\right): \tilde{v}$ $=2960,1758,1368 \mathrm{~cm}^{-1}$. MS $(70 \mathrm{eV}, \mathrm{EI}): \mathrm{m} / z(\%)=618(6)[\mathrm{M}-$ 1] ${ }^{+}, 575$ (35) $\left[\mathrm{M}-\mathrm{C}_{2} \mathrm{H}_{3} \mathrm{O}\right]^{+}, 169$ (100). HRMS (EI): calcd. for $\mathrm{C}_{27} \mathrm{H}_{38} \mathrm{O}_{16}[\mathrm{M}-1]^{+}$618.2160; found 618.2180. $\mathrm{C}_{27} \mathrm{H}_{38} \mathrm{O}_{16}$ (618.58): calcd. C 52.42, H 6.19; found C 52.33, H 6.18 .

Methyl 6,7,8-Trideoxy-2,3-di- $O$-methyl-4- $O$-(2,3,4,6-tetra- $O$ methyl- $\alpha$-D-glucopyranosyl)- $\beta$-D-xylo-oct-7-enopyranoside $\quad(30)$ : Compound $\mathbf{3 0}$ was obtained as a colourless oil in $35 \%$ yield from 29, $(5 R / 5 S, 2.3: 1)$. H NMR: $\delta=2.19(\mathrm{~m}, 1 \mathrm{H}), 2.27-2.40(\mathrm{~m}, 3$ H), $3.00(\mathrm{dd}, J=8.3,7.7 \mathrm{~Hz}, 1 \mathrm{H}), 3.03(\mathrm{dd}, J=9.1,7.6 \mathrm{~Hz}, 1 \mathrm{H})$, $3.16(\mathrm{dd}, J=9.9,4.0 \mathrm{~Hz}, 2 \mathrm{H}), 3.21(\mathrm{dd}, J=9.3,9.3 \mathrm{~Hz}, 2 \mathrm{H}), 3.38$ (s, $6 \mathrm{H}), 3.42(\mathrm{dd}, J=8.5,8.5 \mathrm{~Hz}, 4 \mathrm{H}), 3.42-3.45(\mathrm{~m}, 2 \mathrm{H}), 3.46-$ $3.48(\mathrm{~m}, 2 \mathrm{H}), 3.52(\mathrm{~s}, 6 \mathrm{H}), 3.53(\mathrm{~s}, 6 \mathrm{H}), 3.54(\mathrm{~s}, 12 \mathrm{H}), 3.56(\mathrm{~s}, 6$ H), 3.62 (s, $6 \mathrm{H}), 3.72-3.76$ (m, $2 \mathrm{H}), 3.82-3.85$ (m, $2 \mathrm{H}), 3.90-3.93$ $(\mathrm{m}, 2 \mathrm{H}), 4.17(\mathrm{~d}, J=7.8 \mathrm{~Hz}, 1 \mathrm{H}), 4.19(\mathrm{~d}, J=7.7 \mathrm{~Hz}, 1 \mathrm{H}), 5.12-$ $5.18(\mathrm{~m}, 4 \mathrm{H}), 5.55(\mathrm{~d}, J=2.5 \mathrm{~Hz}, 1 \mathrm{H}), 5.59(\mathrm{~d}, J=4.0 \mathrm{~Hz}, 1 \mathrm{H})$, 5.78-5.88 (m, $2 \mathrm{H}) \mathrm{ppm}$; an NOE correlation was observed between 5- $\mathrm{H}$ and $3-\mathrm{H}$ for the major $(5 R)$ isomer. ${ }^{13} \mathrm{C}$ NMR: $\delta=33.3(2 \times$ $\left.\mathrm{CH}_{2}\right), 55.0\left(\mathrm{CH}_{3}\right), 55.4\left(\mathrm{CH}_{3}\right), 57.1\left(\mathrm{CH}_{3}\right), 57.6\left(\mathrm{CH}_{3}\right), 58.2(2 \times$ $\left.\mathrm{CH}_{3}\right), 58.3\left(2 \times \mathrm{CH}_{3}\right), 58.5\left(2 \times \mathrm{CH}_{3}\right)_{3} 58.8(2 \times \mathrm{CH})_{3} 59.1(2 \times$ $\left.\mathrm{CH}_{3}\right), 68.2(2 \times \mathrm{CH}), 69.2\left(\mathrm{CH}_{2}\right), 69.4(2 \times \mathrm{CH}), 70.1\left(\mathrm{CH}_{2}\right), 73.0$ $(2 \times \mathrm{CH}), 77.7(2 \times \mathrm{CH}), 79.9(2 \times \mathrm{CH}), 81.5(2 \times \mathrm{CH}), 82.6(2 \times$ $\mathrm{CH}), 84.5(2 \times \mathrm{CH}), 95.2(\mathrm{CH}), 95.7(\mathrm{CH}), 102.7(\mathrm{CH}), 102.8(\mathrm{CH})$, $116.1\left(2 \times \mathrm{CH}_{2}\right), 133.2(2 \times \mathrm{CH}) \mathrm{ppm}$. IR $\left(\mathrm{CCl}_{4}\right): \tilde{v}=2933,1445$, $1103 \mathrm{~cm}^{-1}$. MS (70 eV, EI): $\mathrm{m} / z(\%)=450(5)[\mathrm{M}]^{+}, 449(48)[\mathrm{M}-$ $1]^{+}, 88$ (100). HRMS (EI): calcd. for $\mathrm{C}_{21} \mathrm{H}_{38} \mathrm{O}_{10}[\mathrm{M}]^{+} 450.2465$; found 450.2454. $\mathrm{C}_{21} \mathrm{H}_{38} \mathrm{O}_{10}$ (450.52): calcd. C 55.99, $\mathrm{H}$ 8.50; found C 55.71, H 8.42 .

Methyl 7,8,9-Trideoxy-2,3-di- $O$-methyl-4- $O$-(2,3,4,6-tetra- $O$ methyl- $\alpha$-D-glucopyranosyl)-L-glycero- $\beta$-D-gluco-non-8-enopyranoside (31): Compound $\mathbf{3 1}$ was obtained as a colourless oil in $44 \%$ yield from 29. $[\alpha]_{\mathrm{D}}=+59.5\left(c=0.19, \mathrm{CHCl}_{3}\right) .{ }^{1} \mathrm{H} \mathrm{NMR}: \delta=$ 1.64 (br. s, $1 \mathrm{H}), 2.37$ (ddd, $J=14.8,7.1,7.1 \mathrm{~Hz}, 1 \mathrm{H}), 2.45$ (ddd, $J=13.8,7.4,7.4 \mathrm{~Hz}, 1 \mathrm{H}), 3.04(\mathrm{~m}, 1 \mathrm{H}), 3.07(\mathrm{dd}, J=8.2,8.2 \mathrm{~Hz}$, $1 \mathrm{H}), 3.18(\mathrm{dd}, J=9.8,4.2 \mathrm{~Hz}, 1 \mathrm{H}), 3.23(\mathrm{dd}, J=9.8,1.6 \mathrm{~Hz}, 1$ $\mathrm{H}), 3.39(\mathrm{~s}, 3 \mathrm{H}), 3.42(\mathrm{dd}, J=9.0,9.0 \mathrm{~Hz}, 2 \mathrm{H}), 3.48(\mathrm{dd}, J=$ 10.3, $6.4 \mathrm{~Hz}, 1 \mathrm{H}), 3.51(\mathrm{~s}, 3 \mathrm{H}), 3.53(\mathrm{~s}, 3 \mathrm{H}), 3.55$ (s, $3 \mathrm{H}), 3.57$ (s, $3 \mathrm{H}), 3.58(\mathrm{~s}, 3 \mathrm{H}), 3.62-3.68(\mathrm{~m}, 2 \mathrm{H}), 3.64$ (s, $3 \mathrm{H}), 3.87$ (ddd, $J=6.9,6.9,0 \mathrm{~Hz}, 1 \mathrm{H}), 3.93(\mathrm{dd}, J=9.2,9.2 \mathrm{~Hz}, 1 \mathrm{H}), 4.10(\mathrm{~d}, J$ $=7.7 \mathrm{~Hz}, 1 \mathrm{H}), 5.06(\mathrm{dd}, J=18.8,1.9 \mathrm{~Hz}, 1 \mathrm{H}), 5.58(\mathrm{~d}, J=4.2 \mathrm{~Hz}$, $1 \mathrm{H}), 5.79(\mathrm{~m}, 1 \mathrm{H}) \mathrm{ppm}$; the signal for $1 \mathrm{H}$ from an $\mathrm{OH}$ group is missing. ${ }^{13} \mathrm{C}$ NMR: $\delta=38.7\left(\mathrm{CH}_{2}\right), 57.1\left(\mathrm{CH}_{3}\right), 59.6\left(\mathrm{CH}_{3}\right), 60.2$ $\left(\mathrm{CH}_{3}\right), 60.3\left(\mathrm{CH}_{3}\right), 60.5\left(\mathrm{CH}_{3}\right), 60.9\left(\mathrm{CH}_{3}\right), 61.2\left(\mathrm{CH}_{3}\right), 67.7(\mathrm{CH})$, $71.6(\mathrm{CH}), 72.0\left(\mathrm{CH}_{2}\right), 73.2(\mathrm{CH}), 75.0(\mathrm{CH}), 80.5(\mathrm{CH}), 82.2(\mathrm{CH})$, $84.0(\mathrm{CH}), 84.6(\mathrm{CH}), 86.9(\mathrm{CH}), 97.4(\mathrm{CH}), 104.8(\mathrm{CH}), 117.5$ $\left(\mathrm{CH}_{2}\right), 135.4(\mathrm{CH}) \mathrm{ppm}$. IR $\left(\mathrm{CCl}_{4}\right): \tilde{v}=3486,2932,1445 \mathrm{~cm}^{-1}$. MS $\left(\mathrm{FAB}^{+}\right): m / z(\%)=503(82)[\mathrm{M}+\mathrm{Na}]^{+}$. HRMS $\left(\mathrm{FAB}^{+}\right)$: calcd. for $\mathrm{C}_{22} \mathrm{H}_{40} \mathrm{NaO}_{11}$ 503.2468; found 503.2469. $\mathrm{C}_{22} \mathrm{H}_{40} \mathrm{O}_{11}$ (480.55): calcd. C 54.99, H 8.39; found C 55.03, H 8.34.

Methyl 2,3-Di- $O$-methyl-4- $O$-(2,3,4-tri- $O$-acetyl-6,7,8-trideoxy- $\alpha$-Dlyxo-oct-7-enopyranosyl)- $\alpha$-D-glucopyranoside (33): A mixture of isomers $33\left(5^{\prime} S / 5^{\prime} R\right.$ 6.3:1) was obtained as a colourless oil in $29 \%$ yield from 32. ${ }^{1} \mathrm{H}$ NMR: $\delta=1.82$ (br. s, $\left.2 \mathrm{H}\right), 1.98(\mathrm{~s}, 6 \mathrm{H}), 2.03$ (s, $6 \mathrm{H}), 2.13$ (s, $6 \mathrm{H}), 2.29-2.36(\mathrm{~m}, 4 \mathrm{H}), 3.17-3.21(\mathrm{~m}, 2 \mathrm{H}), 3.42$ $(\mathrm{s}, 6 \mathrm{H}), 3.49(\mathrm{~s}, 3 \mathrm{H}), 3.50(\mathrm{~s}, 3 \mathrm{H}), 3.56-3.66(\mathrm{~m}, 6 \mathrm{H}), 3.59$ (s, 6 H), 3.73-3.89 (m, $6 \mathrm{H}), 4.81(\mathrm{~d}, J=3.4 \mathrm{~Hz}, 1 \mathrm{H}), 4.84(\mathrm{~d}, J=$ $3.4 \mathrm{~Hz}, 1 \mathrm{H}), 5.09-5.17(\mathrm{~m}, 6 \mathrm{H}), 5.20(\mathrm{~d}, J=1.8 \mathrm{~Hz}, 2 \mathrm{H}), 5.25$ $(\mathrm{dd}, J=9.8,3.2 \mathrm{~Hz}, 2 \mathrm{H}), 5.30(\mathrm{dd}, J=3.3,2.0 \mathrm{~Hz}, 2 \mathrm{H}), 5.81-$ $5.92(\mathrm{~m}, 2 \mathrm{H})$ ppm. ${ }^{13} \mathrm{C} \mathrm{NMR:} \delta=20.7\left(2 \times \mathrm{CH}_{3}\right), 20.8\left(4 \times \mathrm{CH}_{3}\right)$, $36.1\left(2 \times \mathrm{CH}_{2}\right), 55.2\left(2 \times \mathrm{CH}_{3}\right), 58.9\left(2 \times \mathrm{CH}_{3}\right), 61.2\left(2 \times \mathrm{CH}_{3}\right)$, $61.8\left(2 \times \mathrm{CH}_{2}\right), 69.1(2 \times \mathrm{CH}), 69.4(2 \times \mathrm{CH}), 69.8(2 \times \mathrm{CH}), 70.1$ $(2 \times \mathrm{CH}), 70.7(2 \times \mathrm{CH}), 76.4(2 \times \mathrm{CH}), 82.4(2 \times \mathrm{CH}), 83.1(2 \times$ 
$\mathrm{CH}), 97.4(2 \times \mathrm{CH}), 99.2(2 \times \mathrm{CH}), 117.9\left(2 \times \mathrm{CH}_{2}\right), 133.4(2 \times$ $\mathrm{CH}), 169.8(2 \times \mathrm{C}), 169.9(2 \times \mathrm{C}), 170.0(2 \times \mathrm{C}) \mathrm{ppm}$. IR $\left(\mathrm{CCl}_{4}\right)$ : $\tilde{v}=3606,2930,1754 \mathrm{~cm}^{-1}$. MS $\left(\mathrm{ESI}^{+}\right): \mathrm{m} / z(\%)=543(100)[\mathrm{M}+$ $\mathrm{Na}]^{+}$. HRMS $\left(\mathrm{ESI}^{+}\right)$: calcd. for $\mathrm{C}_{23} \mathrm{H}_{36} \mathrm{NaO}_{13}[\mathrm{M}+\mathrm{Na}]^{+}$543.2054; found 543.2054. $\mathrm{C}_{23} \mathrm{H}_{36} \mathrm{O}_{13}$ (520.52): calcd. C 53.07, $\mathrm{H}$ 6.97; found C 53.27, H 7.19.

Methyl 6- $O$-Allyl-2,3-di- $O$-methyl-4- $O$-(2,3,4-tri- $O$-acetyl- $\alpha$-Dlyxopyranosyl)- $\alpha$-D-glucopyranoside (34): Compound 34 was obtained as a colourless oil in $17 \%$ yield from 32. $[\alpha]_{\mathrm{D}}=+155.0(c=$ 0.34, $\left.\mathrm{CHCl}_{3}\right) .{ }^{1} \mathrm{H}$ NMR: $\delta=2.03(\mathrm{~s}, 3 \mathrm{H}), 2.06$ (s, $\left.3 \mathrm{H}\right), 2.11(\mathrm{~s}, 3$ $\mathrm{H}), 3.23(\mathrm{dd}, J=9.4,3.6 \mathrm{~Hz}, 1 \mathrm{H}), 3.43(\mathrm{~s}, 3 \mathrm{H}), 3.49(\mathrm{~s}, 3 \mathrm{H})$, $3.53-3.71(\mathrm{~m}, 6 \mathrm{H}), 3.60$ (s, $3 \mathrm{H}), 3.88(\mathrm{dd}, J=11.3,5.2 \mathrm{~Hz}, 1 \mathrm{H})$, 4.02-4.04 (m, $2 \mathrm{H}), 4.83$ (d, $J=3.4 \mathrm{~Hz}, 1 \mathrm{H}), 5.14-5.18(\mathrm{~m}, 3 \mathrm{H})$, 5.23-5.34 (m, $3 \mathrm{H}), 5.92$ (dddd, $J=10.3,10.3,10.3,5.6 \mathrm{~Hz}, 1 \mathrm{H}$ ) ppm. ${ }^{13} \mathrm{C} \mathrm{NMR:} \delta=20.7\left(\mathrm{CH}_{3}\right.$, $20.8\left(2 \times \mathrm{CH}_{3}\right.$, $55.3(\mathrm{CH})_{3} 58.8$ $\left(\mathrm{CH}_{3}\right), 60.9\left(\mathrm{CH}_{2}\right), 61.1\left(\mathrm{CH}_{3}\right), 67.1(\mathrm{CH}), 68.5(\mathrm{CH}), 68.6\left(\mathrm{CH}_{2}\right)$, $69.4(\mathrm{CH}), 69.7(\mathrm{CH}), 72.5\left(\mathrm{CH}_{2}\right), 76.5(\mathrm{CH}), 82.4(\mathrm{CH}), 83.2(\mathrm{CH})$, $97.4(\mathrm{CH}), 99.2(\mathrm{CH}), 117.1\left(\mathrm{CH}_{2}\right), 134.7(\mathrm{CH}), 169.8(2 \times \mathrm{C})$, 170.0 (C) ppm. IR $\left(\mathrm{CCl}_{4}\right): \tilde{v}=2931,1753,1223 \mathrm{~cm}^{-1}$. MS $\left(\mathrm{ESI}^{+}\right)$: $m / z(\%)=543(100)[\mathrm{M}+\mathrm{Na}]^{+}$. HRMS $\left(\mathrm{ESI}^{+}\right):$calcd. for $\mathrm{C}_{23} \mathrm{H}_{36} \mathrm{NaO}_{13}$ 543.2054; found 543.2047. $\mathrm{C}_{23} \mathrm{H}_{6} \mathrm{Q}$ (520.52): calcd. C 53.07, H 6.97; found C 53.13, H 7.06.

Methyl 2,3-Di- $O$-methyl-4- $O$-(2,3,5-tri- $O$-acetyl-4- $C$-allyl- $\alpha$-D-arabinofuranosyl)- $\alpha$-D-glucopyranoside (36): Compound 36 was obtained as a colourless oil in $27 \%$ yield from 35. $[\alpha]_{\mathrm{D}}=+112.0(c=$ 0.20, $\left.\mathrm{CHCl}_{3}\right) .{ }^{1} \mathrm{H}$ NMR: $(500 \mathrm{MHz}) \delta=2.06(\mathrm{~s}, 3 \mathrm{H}), 2.11(\mathrm{~s}, 3$ H), $2.14(\mathrm{~s}, 3 \mathrm{H}), 2.34(\mathrm{dd}, J=7.5,0 \mathrm{~Hz}, 2 \mathrm{H}), 3.20-3.23(\mathrm{~m}, 1 \mathrm{H})$, $3.42(\mathrm{~s}, 3 \mathrm{H}), 3.46-3.51(\mathrm{~m}, 2 \mathrm{H}), 3.51(\mathrm{~s}, 3 \mathrm{H}), 3.56(\mathrm{~s}, 3 \mathrm{H}), 3.70$ $(\mathrm{m}, 1 \mathrm{H}), 4.06(\mathrm{~d}, J=6.3 \mathrm{~Hz}, 1 \mathrm{H}), 4.16(\mathrm{~d}, J=12.0 \mathrm{~Hz}, 1 \mathrm{H}), 4.28$ $(\mathrm{d}, J=12.0 \mathrm{~Hz}, 1 \mathrm{H}), 4.29(\mathrm{dd}, J=12.0,0 \mathrm{~Hz}, 1 \mathrm{H}), 4.35(\mathrm{dd}, J=$ $12.0,5.0 \mathrm{~Hz}, 1 \mathrm{H}), 4.82(\mathrm{~d}, J=3.5 \mathrm{~Hz}, 1 \mathrm{H}), 5.11(\mathrm{dd}, J=6.3$, $4.1 \mathrm{~Hz}, 2 \mathrm{H}), 5.11(\mathrm{dd}, J=10.4,0 \mathrm{~Hz}, 1 \mathrm{H}), 5.16(\mathrm{dd}, J=17.0$, $0 \mathrm{~Hz}, 1 \mathrm{H}), 5.35(\mathrm{~d}, J=3.8 \mathrm{~Hz}, 1 \mathrm{H}), 5.77$ (dddd, $J=17.4,10.4$, $7.5,7.5 \mathrm{~Hz}, 1 \mathrm{H}) \mathrm{ppm}$; an NOE correlation was observed between $6^{\prime}-\mathrm{H}$ and $2^{\prime}-\mathrm{H} .{ }^{13} \mathrm{C}$ NMR $(125.7 \mathrm{MHz}): \delta=20.8\left(2 \times \mathrm{CH}_{3}\right), 21.1$ $\left(\mathrm{CH}_{3}\right), 39.9\left(\mathrm{CH}_{2}\right), 55.3\left(\mathrm{CH}_{3}\right), 58.9\left(\mathrm{CH}_{3}\right), 61.1\left(\mathrm{CH}_{3}\right), 63.2\left(\mathrm{CH}_{2}\right)$, $65.5\left(\mathrm{CH}_{2}\right), 68.2(\mathrm{CH}), 77.4(\mathrm{CH}), 78.2(\mathrm{CH}), 81.9(\mathrm{CH}), 83.0(\mathrm{CH})$, $85.7(\mathrm{CH}), 85.9(\mathrm{C}) 97.3(\mathrm{CH}), 106.3(\mathrm{CH}), 119.5\left(\mathrm{CH}_{2}\right), 132.1$ $(\mathrm{CH}), 170.6(2 \times \mathrm{C}), 172.1(\mathrm{C}) \mathrm{ppm}$. IR $\left(\mathrm{CCl}_{4}\right): \tilde{v}=3519,3421$, 1747, $1242 \mathrm{~cm}^{-1}$. MS $\left(\mathrm{ESI}^{+}\right): \mathrm{m} / z(\%)=543(100)[\mathrm{M}+\mathrm{Na}]^{+}$. HRMS $\left(\mathrm{ESI}^{+}\right)$: calcd. for $\mathrm{C}_{2} \mathrm{H}$ 36aO $[\mathrm{MM}+\mathrm{Na}]^{+} 543.2054$; found 543.2056. $\mathrm{C}_{23} \mathrm{H}_{36} \mathrm{O}_{13}$ (520.52): calcd. C 53.07, H 6.97; found $\mathrm{C}$ 53.23, H 7.01.

Methyl 2,3-Di- $O$-methyl-4- $O$-(2,3,5-tri- $O$-acetyl-4- $C$-allyl- $\beta$-L-xylofuranosyl)- $\alpha$-D-glucopyranoside (37): Compound $\mathbf{3 7}$ was obtained as a colourless oil in $25 \%$ yield from 35. $[\alpha]_{\mathrm{D}}=+132.8(c=0.29$, $\left.\mathrm{CHCl}_{3}\right) .{ }^{1} \mathrm{H}$ NMR $(500 \mathrm{MHz}): \delta=2.05(\mathrm{~s}, 3 \mathrm{H}), 2.08(\mathrm{~s}, 3 \mathrm{H}), 2.14$ (s, $3 \mathrm{H}), 2.36-2.44(\mathrm{~m}, 2 \mathrm{H}), 3.20(\mathrm{dd}, J=9.3,3.6 \mathrm{~Hz}, 1 \mathrm{H}), 3.40$ (s, $3 \mathrm{H}), 3.50(\mathrm{~s}, 3 \mathrm{H}), 3.52(\mathrm{~s}, 3 \mathrm{H}), 3.52(\mathrm{dd}, J=9.4,5.0 \mathrm{~Hz}, 1 \mathrm{H})$, $3.55-3.61(\mathrm{~m}, 2 \mathrm{H}), 3.73(\mathrm{dd}, J=12.4,2.0 \mathrm{~Hz}, 1 \mathrm{H}), 3.90(\mathrm{dd}, J=$ $12.6,3.1 \mathrm{~Hz}, 1 \mathrm{H}), 4.12(\mathrm{~d}, J=12.0 \mathrm{~Hz}, 1 \mathrm{H}), 4.23(\mathrm{~d}, J=12.0 \mathrm{~Hz}$, $1 \mathrm{H}), 4.81(\mathrm{~d}, J=3.5 \mathrm{~Hz}, 1 \mathrm{H}), 5.13-5.21(\mathrm{~m}, 2 \mathrm{H}), 5.32(\mathrm{~d}, J=$ $6.0 \mathrm{~Hz}, 1 \mathrm{H}), 5.41(\mathrm{~d}, J=6.0 \mathrm{~Hz}, 1 \mathrm{H}), 5.44(\mathrm{~d}, J=4.1 \mathrm{~Hz}, 1 \mathrm{H})$, 5.75 (dddd, $J=17.3,10.1,7.2,7.2 \mathrm{~Hz}, 1 \mathrm{H}$ ) ppm; the signal for 1 $\mathrm{H}$ from an $\mathrm{OH}$ group is missing; an NOE correlation was observed between 6 '-H and 3 '-H. ${ }^{13} \mathrm{C}$ NMR $(125.7 \mathrm{MHz}): \delta=20.7\left(\mathrm{CH}_{3}\right)$, $20.8\left(\mathrm{CH}_{3}\right), 20.9\left(\mathrm{CH}_{3}\right), 39.9\left(\mathrm{CH}_{2}\right), 55.2\left(\mathrm{CH}_{3}\right), 59.0\left(\mathrm{CH}_{3}\right), 61.0$ $\left(\mathrm{CH}_{3}\right), 61.3\left(\mathrm{CH}_{2}\right), 65.1\left(\mathrm{CH}_{2}\right), 70.3(\mathrm{CH}), 76.6(\mathrm{CH}), 77.1(\mathrm{CH})$, $80.0(\mathrm{CH}), 82.2(\mathrm{CH}), 83.0(\mathrm{CH}), 84.5(\mathrm{C}), 97.6(\mathrm{CH}), 106.2(\mathrm{CH})$, $120.5\left(\mathrm{CH}_{2}\right), 130.9(\mathrm{CH}), 169.6(\mathrm{C}), 169.8(\mathrm{C}), 170.4(\mathrm{C}) \mathrm{ppm}$. IR $\left(\mathrm{CCl}_{4}\right): \tilde{v}=3520,3551,2932,1752 \mathrm{~cm}^{-1}$. MS $\left(\mathrm{ESI}^{+}\right): \mathrm{m} / \mathrm{z}(\%)=543$ (100) $[\mathrm{M}+\mathrm{Na}]^{+}$. HRMS $\left(\mathrm{ESI}^{+}\right)$: calcd. for $\mathrm{C}_{23} \mathrm{H}_{36} \mathrm{NaO}_{13}[\mathrm{M}+$
$\mathrm{Na}]^{+}$543.2054; found 543.2061. $\mathrm{C}_{23} \mathrm{H}_{36} \mathrm{O}_{13}$ (520.52): calcd. $\mathrm{C}$ 53.07, H 6.97; found C 53.17, H 6.99.

Methyl 2,3-Di- $O$-methyl-4-O-(4-C -allyl-2,3,5-tri- $O$-methyl- $\beta$-L-xylofuranosyl)- $\alpha$-D-glucopyranoside (39): Compound $\mathbf{3 9}$ was obtained as a colourless oil in $36 \%$ yield from 38. $[\alpha]_{\mathrm{D}}=+61.3(c=0.43$, $\left.\mathrm{CHCl}_{3}\right) .{ }^{1} \mathrm{H}$ NMR: $(500 \mathrm{MHz}) \delta=2.31(\mathrm{dd}, J=14.2,7.9 \mathrm{~Hz}, 1$ H), $2.41(\mathrm{dd}, J=14.2,6.6 \mathrm{~Hz}, 1 \mathrm{H}), 3.24(\mathrm{dd}, J=9.5,3.8 \mathrm{~Hz}, 1$ $\mathrm{H}), 3.35(\mathrm{~d}, J=10.1 \mathrm{~Hz}, 1 \mathrm{H}), 3.36(\mathrm{~s}, 3 \mathrm{H}), 3.40(\mathrm{~s}, 3 \mathrm{H}), 3.42(\mathrm{~s}$, $3 \mathrm{H}), 3.45(\mathrm{~d}, J=10.1 \mathrm{~Hz}, 1 \mathrm{H}), 3.48(\mathrm{~s}, 3 \mathrm{H}), 3.49$ (s, $3 \mathrm{H}), 3.52-$ $3.56(\mathrm{~m}, 2 \mathrm{H}), 3.59(\mathrm{~s}, 3 \mathrm{H}), 3.63(\mathrm{~d}, J=6.3 \mathrm{~Hz}, 1 \mathrm{H}), 3.67(\mathrm{dd}, J$ $=12.3,2.2 \mathrm{~Hz}, 2 \mathrm{H}), 3.84(\mathrm{dd}, J=6.5,4.3 \mathrm{~Hz}, 1 \mathrm{H}), 3.98(\mathrm{dd}, J=$ 12.6, $2.5 \mathrm{~Hz}, 1 \mathrm{H}), 4.82(\mathrm{~d}, J=3.5 \mathrm{~Hz}, 1 \mathrm{H}), 5.07-5.15(\mathrm{~m}, 2 \mathrm{H})$, $5.30(\mathrm{~d}, J=4.1 \mathrm{~Hz}, 1 \mathrm{H}), 5.77(\mathrm{~m}, 1 \mathrm{H}) \mathrm{ppm}$; the signal for $1 \mathrm{H}$ from an $\mathrm{OH}$ group is missing; NOE correlations were observed between $6{ }^{\prime}-\mathrm{H}$ and $3^{\prime}-\mathrm{H}$, and between $5^{\prime}-\mathrm{H}$ and $2^{\prime}-\mathrm{H} .{ }^{13} \mathrm{C}$ NMR $(125.7 \mathrm{MHz}): \delta=40.2\left(\mathrm{CH}_{2}\right), 55.0\left(\mathrm{CH}_{3}\right), 58.1\left(\mathrm{CH}_{3}\right), 58.6\left(\mathrm{CH}_{3}\right)$, $58.9\left(\mathrm{CH}_{3}\right), 59.1\left(\mathrm{CH}_{3}\right), 60.8\left(\mathrm{CH}_{3}\right), 61.2\left(\mathrm{CH}_{2}\right), 70.6(\mathrm{CH}), 74.2$ $\left(\mathrm{CH}_{2}\right), 75.2(\mathrm{CH}), 82.6(\mathrm{CH}), 83.2(\mathrm{CH}), 85.1(\mathrm{C}), 86.5(\mathrm{CH}), 89.0$ $(\mathrm{CH}), 97.4(\mathrm{CH}), 106.9(\mathrm{CH}), 118.9\left(\mathrm{CH}_{2}\right), 132.9(\mathrm{CH}) \mathrm{ppm}$. IR $\left(\mathrm{CCl}_{4}\right): \tilde{v}=3517,2929,1053 \mathrm{~cm}^{-1}$. MS $\left(\mathrm{ESI}^{+}\right): \mathrm{m} / \mathrm{z}(\%)=459(100)$ $[\mathrm{M}+\mathrm{Na}]^{+}$. HRMS $\left(\mathrm{ESI}^{+}\right)$: calcd. for $\mathrm{C}_{20} \mathrm{H}_{36} \mathrm{NaO}_{10}[\mathrm{M}+\mathrm{Na}]^{+}$ 459.2206; found 459.2209. $\mathrm{C}_{20} \mathrm{H}_{36} \mathrm{O}_{10}$ (436.49): calcd. C 55.03, $\mathrm{H}$ 8.31; found C 55.07, H 8.05.

Methyl 2,3-Di- $O$-methyl-4- $O$-(4- $C$-allyl-2,3,5-tri- $O$-methyl- $\alpha$-Darabinofuranosyl)- $\alpha$-D-glucopyranoside (40): Compound $\mathbf{4 0}$ was obtained as a colourless oil in $15 \%$ yield from 38. $[\alpha]_{\mathrm{D}}=+42.1(c=$ $\left.0.33, \mathrm{CHCl}_{3}\right) .{ }^{1} \mathrm{H}$ NMR $(500 \mathrm{MHz}): \delta=2.42(\mathrm{dd}, J=14.5,7.9 \mathrm{~Hz}$, $1 \mathrm{H}), 2.48(\mathrm{dd}, J=14.1,6.3 \mathrm{~Hz}, 1 \mathrm{H}), 3.21(\mathrm{dd}, J=9.1,3.5 \mathrm{~Hz}, 1$ $\mathrm{H}), 3.26(\mathrm{~d}, J=9.8 \mathrm{~Hz}, 1 \mathrm{H}), 3.30(\mathrm{~d}, J=9.8 \mathrm{~Hz}, 1 \mathrm{H}), 3.34(\mathrm{~s}, 3$ H), 3.40 (s, $3 \mathrm{H}), 3.42(\mathrm{~s}, 3 \mathrm{H}), 3.48(\mathrm{~s}, 3 \mathrm{H}), 3.49$ (s, $3 \mathrm{H}), 3.52$ $3.60(\mathrm{~m}, 3 \mathrm{H}), 3.59$ (s, $3 \mathrm{H}), 3.68-3.73(\mathrm{~m}, 3 \mathrm{H}), 3.94(\mathrm{dd}, J=12.6$, $2.8 \mathrm{~Hz}, 1 \mathrm{H}), 4.81(\mathrm{~d}, J=3.8 \mathrm{~Hz}, 1 \mathrm{H}), 5.10-5.15(\mathrm{~m}, 2 \mathrm{H}), 5.31$ $(\mathrm{d}, J=3.1 \mathrm{~Hz}, 1 \mathrm{H}), 5.85(\mathrm{~m}, 1 \mathrm{H}) \mathrm{ppm}$; the signal for $1 \mathrm{H}$ from an $\mathrm{OH}$ group is missing; an NOE correlation was observed between $5^{\prime}-\mathrm{H}$ and $11^{\prime}-\mathrm{H} .{ }^{13} \mathrm{C} \mathrm{NMR}: \delta=37.2\left(\mathrm{CH}_{2}\right), 55.1\left(\mathrm{CH}_{3}\right), 58.0\left(\mathrm{CH}_{3}\right)$, $58.6\left(\mathrm{CH}_{3}\right), 58.8\left(\mathrm{CH}_{3}\right), 59.3\left(\mathrm{CH}_{3}\right), 60.9\left(\mathrm{CH}_{3}\right), 61.9\left(\mathrm{CH}_{2}\right), 70.4$ $(\mathrm{CH}), 74.9\left(\mathrm{CH}_{2}\right), 76.3(\mathrm{CH}), 82.5(\mathrm{CH}), 83.1(\mathrm{CH}), 85.1(\mathrm{C}), 86.2$ $(\mathrm{CH}), 88.7(\mathrm{CH}), 97.4(\mathrm{CH}), 106.9(\mathrm{CH}), 118.4\left(\mathrm{CH}_{2}\right), 133.4(\mathrm{CH})$ ppm. IR $\left(\mathrm{CCl}_{4}\right): \tilde{v}=3518,2931,1055 \mathrm{~cm}^{-1}$. MS $\left(\mathrm{ESI}^{+}\right): \mathrm{m} / \mathrm{z}(\%)=$ 459 (100) $[\mathrm{M}+\mathrm{Na}]^{+}$. HRMS $\left(\mathrm{ESI}^{+}\right)$: calcd. for $\mathrm{C}_{20} \mathrm{H}_{36} \mathrm{NaO}_{10}[\mathrm{M}$ $+\mathrm{Na}^{+}$459.2206; found 459.2212. $\mathrm{C}_{20} \mathrm{H}_{6}{ }_{1} \oint$ (436.49): calcd. C $55.03, \mathrm{H}$ 8.31; found C 55.18, H 8.03.

Methyl 6-O-Allyl-2,3-di- $O$-methyl-4- $O$-(2,3,5-tri- $O$-methyl- $\alpha$-Darabinofuranosyl)- $\alpha$-D-glucopyranoside (41): Compound 41 was obtained as an amorphous solid in $21 \%$ yield from 38. $[\alpha]_{\mathrm{D}}=+56.2$ $\left(c=0.38, \mathrm{CHCl}_{3}\right) .{ }^{1} \mathrm{H}$ NMR $(500 \mathrm{MHz}): \delta=3.24(\mathrm{dd}, J=9.5,3.5$ $\mathrm{Hz}, 1 \mathrm{H}), 3.29(\mathrm{~s}, 3 \mathrm{H}), 3.40(\mathrm{~s}, 3 \mathrm{H}), 3.41(\mathrm{~s}, 3 \mathrm{H}), 3.43$ (s, 3 H), $3.49(\mathrm{~s}, 3 \mathrm{H}), 3.52(\mathrm{dd}, J=5.0,0 \mathrm{~Hz}, 2 \mathrm{H}), 3.54-3.62(\mathrm{~m}, 3 \mathrm{H})$, $3.58(\mathrm{~s}, 3 \mathrm{H}), 3.70-3.72(\mathrm{~m}, 2 \mathrm{H}), 3.75(\mathrm{dd}, J=2.2,0 \mathrm{~Hz}, 1 \mathrm{H})$, 4.01 (dd, $J=12.6,5.7 \mathrm{~Hz}, 1 \mathrm{H}), 4.07(\mathrm{~m}, 2 \mathrm{H}), 4.09$ (dd, $J=10.7$, $5.0 \mathrm{~Hz}, 1 \mathrm{H}), 4.84(\mathrm{~d}, J=3.5 \mathrm{~Hz}, 1 \mathrm{H}), 5.15(\mathrm{dd}, J=10.4,1.5 \mathrm{~Hz}$, $1 \mathrm{H}), 5.27$ (dd, $J=17.0,1.5 \mathrm{~Hz}, 1 \mathrm{H}), 5.40(\mathrm{~d}, J=0 \mathrm{~Hz}, 1 \mathrm{H}), 5.94$ (dddd, $J=17.2, \quad 10.3,5.6,5.6 \mathrm{~Hz}, 1 \mathrm{H}) \mathrm{ppm} .{ }^{13} \mathrm{C} \mathrm{NMR}$ $(125.7 \mathrm{MHz}): \delta=55.0\left(\mathrm{CH}_{3}\right), 57.5\left(\mathrm{CH}_{3}\right), 58.0\left(\mathrm{CH}_{3}\right), 58.6\left(\mathrm{CH}_{3}\right)$, $59.3\left(\mathrm{CH}_{3}\right), 60.8\left(\mathrm{CH}_{3}\right), 69.0(\mathrm{CH}), 69.6(\mathrm{CH}), 72.4\left(\mathrm{CH}_{2}\right), 72.8$ $\left(\mathrm{CH}_{2}\right), 74.7(\mathrm{CH}), 81.4\left(\mathrm{CH}_{2}\right), 82.2(\mathrm{CH}), 83.3(\mathrm{CH}), 86.3(\mathrm{CH})$, $89.8(\mathrm{CH}), 97.1(\mathrm{CH}), 106.7(\mathrm{CH}), 116.8\left(\mathrm{CH}_{2}\right), 135.0(\mathrm{CH}) \mathrm{ppm}$. IR $\left(\mathrm{CCl}_{4}\right): \tilde{v}=2931,2360,1054 \mathrm{~cm}^{-1} . \mathrm{MS}\left(\mathrm{ESI}^{+}\right): \mathrm{m} / \mathrm{z}(\%)=459$ $(100)\left[\mathrm{M}+\mathrm{Na}^{+}\right.$. HRMS $\left(\mathrm{ESI}^{+}\right)$: calcd. for $\mathrm{C} \mathrm{H} \mathrm{NaO}_{20} \quad 36 \quad 10$ $[\mathrm{M}+\mathrm{Na}]^{+} 459.2206$; found 459.2214. $\mathrm{C}_{20} \mathrm{H}_{36} \mathrm{O}_{10}$ (436.49): calcd. C 55.03, H 8.31; found C 55.23, H 8.43.

Methyl 2,3-Di- $O$-methyl-4- $O$-(4- $C$-allyl-2,3,5,6-tetra- $O$-methyl- $\alpha$-Dmannofuranosyl)- $\alpha$-D-glucopyranoside (43): A mixture of isomers 43 
was obtained as a colourless oil in $52 \%$ yield $\left(4^{\prime} R / 4^{\prime} S, 1: 1\right)$ from 42. ${ }^{1} \mathrm{H}$ NMR: $\delta=2.33(\mathrm{~m}, 1 \mathrm{H}), 2.47(\mathrm{dd}, J=13.5,7.4 \mathrm{~Hz}, 1 \mathrm{H})$, 2.54-2.62 (m, $2 \mathrm{H}), 3.22(\mathrm{dd}, J=9.1,3.6 \mathrm{~Hz}, 1 \mathrm{H}), 3.24(\mathrm{dd}, J=$ 9.0, 3.4 Hz, $1 \mathrm{H}), 3.30(\mathrm{dd}, J=5.4,2.8 \mathrm{~Hz}, 1 \mathrm{H}), 3.35-3.75(\mathrm{~m}, 16$ H), $3.35(\mathrm{~s}, 6 \mathrm{H}), 3.39(\mathrm{~s}, 6 \mathrm{H}), 3.44(\mathrm{~s}, 3 \mathrm{H}), 3.45(\mathrm{~s}, 3 \mathrm{H}), 3.48(\mathrm{~s}$, $3 \mathrm{H}), 3.49$ (s, $6 \mathrm{H}), 3.50(\mathrm{~s}, 6 \mathrm{H}), 3.51(\mathrm{~s}, 3 \mathrm{H}), 3.59(\mathrm{~s}, 3 \mathrm{H}), 3.60$ $(\mathrm{s}, 3 \mathrm{H}), 3.97(\mathrm{~m}, 1 \mathrm{H}), 4.01(\mathrm{~d}, J=6.1 \mathrm{~Hz}, 2 \mathrm{H}), 4.82(\mathrm{~d}, J=$ $3.7 \mathrm{~Hz}, 2 \mathrm{H}), 5.01-5.14(\mathrm{~m}, 4 \mathrm{H}), 5.32(\mathrm{~d}, J=5.3 \mathrm{~Hz}, 1 \mathrm{H}), 5.40$ $(\mathrm{d}, J=2.9 \mathrm{~Hz}, 1 \mathrm{H}), 5.79-5.95(\mathrm{~m}, 2 \mathrm{H}) \mathrm{ppm}$; the signals for $2 \mathrm{H}$ from $\mathrm{OH}$ groups are missing. ${ }^{13} \mathrm{C}$ NMR: $\delta=37.8\left(\mathrm{CH}_{2}\right), 39.6$ $\left(\mathrm{CH}_{2}\right), 55.1\left(2 \times \mathrm{CH}_{3}\right), 58.4\left(2 \times \mathrm{CH}_{3}\right), 58.7\left(\mathrm{CH}_{3}\right), 58.8\left(\mathrm{CH}_{3}\right)$, $59.0\left(\mathrm{CH}_{3}\right), 59.1\left(\mathrm{CH}_{3}\right), 59.3\left(\mathrm{CH}_{3}\right), 59.4\left(\mathrm{CH}_{3}\right), 60.2\left(\mathrm{CH}_{3}\right), 60.4$ $\left(\mathrm{CH}_{3}\right), 60.9\left(\mathrm{CH}_{3}\right), 61.0\left(\mathrm{CH}_{2}\right), 61.1\left(\mathrm{CH}_{2}\right), 61.3\left(\mathrm{CH}_{3}\right), 70.4(\mathrm{CH})$, $70.6(\mathrm{CH}), 71.6\left(\mathrm{CH}_{2}\right), 73.1\left(\mathrm{CH}_{2}\right), 76.5(\mathrm{CH}), 77.9(\mathrm{CH}), 79.8$ $(\mathrm{CH}), 79.9(\mathrm{CH}), 82.0(\mathrm{CH}), 82.2(\mathrm{CH}), 82.3(2 \times \mathrm{CH}), 82.9(\mathrm{CH})$, $83.0(\mathrm{CH}), 83.2(\mathrm{CH}), 84.4(\mathrm{CH}), 87.3(2 \times \mathrm{C}), 97.5(\mathrm{CH}), 97.6$ $(\mathrm{CH}), 106.0(\mathrm{CH}), 107.2(\mathrm{CH}), 117.6\left(\mathrm{CH}_{2}\right), 118.3\left(\mathrm{CH}_{2}\right), 133.7$ $(\mathrm{CH}), 134.4(\mathrm{CH}) \mathrm{ppm}$. IR $\left(\mathrm{CCl}_{4}\right): \tilde{v}=3507,2931,1101 \mathrm{~cm}^{-1}$. MS $\left(\mathrm{ESI}^{+}\right): \mathrm{m} / \mathrm{z}(\%)=503(100)[\mathrm{M}+\mathrm{Na}]^{+}$. HRMS $\left(\mathrm{ESI}^{+}\right):$calcd. for $\mathrm{C}_{22} \mathrm{H}_{40} \mathrm{NaO}_{11}[\mathrm{M}+\mathrm{Na}]^{+}$503.2468; found 503.2459. $\mathrm{C}_{22} \mathrm{H}_{40} \mathrm{O}_{11}$ (480.56): calcd. C 54.99, H 8.39; found C 55.12, H 8.53.

Methyl 6- $O$-Allyl-2,3-di- $O$-methyl-4- $O$-(2,3,5,6-tetra- $O$-methyl- $\alpha$-Dmannofuranosyl)- $\alpha$-D-glucopyranoside (44): Compound 44 was obtained as a colourless oil in $22 \%$ yield from $42 .[\alpha]_{\mathrm{D}}=+67.5(c=$ $\left.0.28, \mathrm{CHCl}_{3}\right) .{ }^{1} \mathrm{H} \mathrm{NMR}: \delta=3.21(\mathrm{dd}, J=9.4,3.6 \mathrm{~Hz}, 1 \mathrm{H}), 3.37-$ $3.62(\mathrm{~m}, 6 \mathrm{H}), 3.38(\mathrm{~s}, 3 \mathrm{H}), 3.42(\mathrm{~s}, 3 \mathrm{H}), 3.45(\mathrm{~s}, 3 \mathrm{H}), 3.48$ (s, 3 $\mathrm{H}), 3.49(\mathrm{~s}, 3 \mathrm{H}), 3.53(\mathrm{~s}, 3 \mathrm{H}), 3.57(\mathrm{~s}, 3 \mathrm{H}), 3.67-3.74(\mathrm{~m}, 3 \mathrm{H})$, $3.92(\mathrm{dd}, J=3.7,3.7 \mathrm{~Hz}, 1 \mathrm{H}), 4.01-4.07(\mathrm{~m}, 3 \mathrm{H}), 4.82(\mathrm{~d}, J=$ $3.4 \mathrm{~Hz}, 1 \mathrm{H}$ ), 5.15 (dddd, $J=10.3,1.3,1.3,1.3 \mathrm{~Hz}, 1 \mathrm{H}$ ), 5.27 (dddd, $J=17.2,1.6,1.6,1.6 \mathrm{~Hz}, 1 \mathrm{H}), 5.38(\mathrm{~d}, J=3.7 \mathrm{~Hz}, 1 \mathrm{H})$, 5.92 (dddd, $J=17.2,10.3,5.6,5.6 \mathrm{~Hz}, 1 \mathrm{H}$ ) ppm. ${ }^{13} \mathrm{C}$ NMR: $\delta=$ $55.1\left(\mathrm{CH}_{3}\right), 57.6\left(\mathrm{CH}_{3}\right), 58.6\left(\mathrm{CH}_{3}\right), 58.7\left(\mathrm{CH}_{3}\right), 59.3\left(\mathrm{CH}_{3}\right), 60.1$ $\left(\mathrm{CH}_{3}\right), 61.0\left(\mathrm{CH}_{3}\right), 69.7(\mathrm{CH}), 69.7\left(\mathrm{CH}_{2}\right), 71.3\left(\mathrm{CH}_{2}\right), 72.2\left(\mathrm{CH}_{2}\right)$, $75.4(\mathrm{CH}), 77.2(\mathrm{CH}), 77.8(\mathrm{CH}), 80.0(\mathrm{CH}), 82.2(\mathrm{CH}), 83.4(\mathrm{CH})$ $87.3(\mathrm{CH}), 97.1(\mathrm{CH}), 106.4(\mathrm{CH}), 116.6\left(\mathrm{CH}_{2}\right), 135.0(\mathrm{CH}) \mathrm{ppm}$. IR $\left(\mathrm{CCl}_{4}\right): \tilde{v}=2929,1708,1064 \mathrm{~cm}^{-1}$. MS $\left(\mathrm{ESI}^{+}\right): \mathrm{m} / \mathrm{z}(\%)=503$ (100) $[\mathrm{M}+\mathrm{Na}]^{+}$. HRMS $\left(\mathrm{ESI}^{+}\right)$: calcd. for $\mathrm{C}_{22} \mathrm{H}_{40} \mathrm{O}_{11} \mathrm{Na}[\mathrm{M}+$ $\mathrm{Na}]^{+}$503.2468; found 503.2460. $\mathrm{C}_{22} \mathrm{H}_{40} \mathrm{O}_{11}$ (480.55): calcd. C 54.99, H 8.39; found C 55.03, H 8.66.

Supporting Information (see footnote on the first page of this article): Complete description of the experimental details of precursors and analytical data for all new compounds.

\section{Acknowledgments}

This work was supported by the Research Programs of the Gobierno de Canarias (SolSubC200801000192 and CTQ2010-18244) of the Ministerio de Ciencia e Innovación, cofinanced by the Fondo Europeo de Desarrollo Regional (FEDER). I. P.-M. thanks the Spanish Ministerio de Ciencia e Innovación for the Research Program PIE 200930I138. L. M. Q. thanks the Consejo Superior de Investigaciones Científicas (CSIC) (Program I3P) for fellowships.

1 a) I. Pérez-Martín, E. Suárez in Radicals and Carbohydrates Encyclopedia of Radicals in Chemistry, Biology and Materials (Eds.: C. Chatgilialoglu, A. Studer), John Wiley \& Sons Ltd, Chichester, 2012, vol. 2, pp. 1131-1174; b) A. J. Pearce, J.-M. Mallet, P. Sinaÿ in Radicals in Organic Synthesis vol. 2 (Eds.: P. Renaud, M. Sibi), Wiley-VCH, Weinheim, 2001, pp. 538577.

2 R. J. Ferrier, J. O. Hoberg, Adv. Carbohydr. Chem. Biochem. 2003, 58, 55-119.

3 C. Taillefumier, Y. Chapleur, Chem. Rev. 2004, 104, 263-292.
4 S. G. Hansen, T. Skrydstrup, Top. Curr. Chem. 2006, 264, 135162.

5 D. E. Levy, P. Fügedi, The Organic Chemistry of Sugars, CRC Taylor \& Francis, Boca Raton, 2006.

6 O. Arjona, A. M. Gómez, J. C. López, J. Plumet, Chem. Rev. 2007, 107, 1919-2036.

7 B. Fraser-Reid, J. C. López, Curr. Org. Chem. 2009, 13, 532553.

8 For general recent reports on intramolecular HAT/C-C bond formation, see: a) A. Baralle, A. Baroudi, M. Daniel, L. Fensterbank, J.-P. Goddard, E. Lacote, M.-H. Larraufie, G. Maestri, M. Malacria, C. Ollivier in Radical Cascade Reactions Encyclopedia of Radicals in Chemistry, Biology and Materials (Eds.: C. Chatgilialoglu, A. Studer), John Wiley \& Sons Ltd, Chichester, 2012, vol. 2; b) G. J. Rowlands, Tetrahedron 2010, 66, 1593-1636; c) R. Jahjah, A. Gassama, V.Bulach, C. Suzuki, M. Abe, N. Hoffmann, A. Martínez, J.-M. Nuzillard, Chem. Eur. J. 2010, 16, 3341-3354; d) M. Albert, L. Fensterbank, E. Lacôte, M. Malacria, Top. Curr. Chem. 2006, 264, 1-62; e) Z. Cekovic, J. Serb. Chem. Soc. 2005, 70, 287-318; f) S. Z. Zard in Radical Reactions in Organic Synthesis (Eds.: R. G. Compton, S. G. Davies, J. Evans), Oxford University Press Inc., New York, 2003, pp. 75-79; g) L. Feray, N. Kuznetsov, P. Renaud in Radicals in Organic Synthesis vol. 2 (Eds.: P. Renaud, M. Sibi), Wiley-VCH, Weinheim, 2001, pp. 246-278; h) J. Robert- son, J. Pillai, R. K. Lush, Chem. Soc. Rev. 2001, 30, 94-103; i) G. Majetich, Tetrahedron 1995, 51, 7095-7129.

[9] a) E. I. León, A. Martín, I. Pérez-Martín, L. M. Quintanal, E. Suárez, Eur. J. Org. Chem. 2010, 5248-5262; b) C. G. Francisco, R. Freire, A. J. Herrera, I. Pérez-Martín, E. Suárez, Tetrahedron 2007, 63, 8910-8920; c) C. G. Francisco, A. J. Herrera, E. Suárez, J. Org. Chem. 2002, 67, 7439-7445; d) C. Chatgilialoglu, T. Gimisis, G. P. Spada, Chem. Eur. J. 1999, 5, 2866-2876; e) R. L. Dorta, A. Martín, J. A. Salazar, E. Suárez, J. Org. Chem. 1998, 63, 2251-2261; f) G. Descotes, J. Carbohydr. Chem. 1988, 7, 1-20. For an interesting synthesis of natural products containing spiroketals by radical HAT on non-carbohydrate systems, see: g) J. Sperry, Y.-C. (W.) Liu, M. A. Brimble, Org. Biomol. Chem. 2010, 8, 29-38.

10 For tandem intramolecular alkoxyl radical promoted HAT/C$\mathrm{C}$ bond formation reactions in carbohydrate models, see: a) A. Martín, I. Pérez-Martín, L. M. Quintanal, E. Suárez, Tetrahedron Lett. 2008, 49, 5179-5181; b) A. J. Herrera, M. Rondón, E. Suárez, J. Org. Chem. 2008, 73, 3384-3391; c) M. Stark, J. Thiem, Carbohydr. Res. 2006, 341, 1543-1556; d) J. C. López, B. Fraser-Reid, Chem. Commun. 1997, 2251-2257; e) J. C. López, R. Alonso, B. Fraser-Reid, J. Am. Chem. Soc. 1989, 111, 6471-6473.

11 For $O$-centered radical-promoted HAT/C-C bond-forming reactions on non-carbohydrate substrates, see: a) H. Zhu, J. G. Wickenden, N. E. Campbell, J. C. T. Leung, K. M. Johnson, G. M. Sammis, Org. Lett. 2009, 11, 2019-2022; b) G. Petrovic, Z. Cekovic, Synthesis 2004, 1671-1679; c) G. Petrovic, Z. Cekovic, Org. Lett. 2000, 2, 3769-3772; d) G. Petrovic, Z. Cekovic, Tetrahedron 1999, 55, 1377-1390; e) G. Petrovic, Z. Cekovic, Tetrahedron Lett. 1997, 38, 627-630; f) Z. Cekovic, D. Ilijev, Tetrahedron Lett. 1988, 29, 1441-1444.

12 For some recent reviews on oxygen-centered radicals, see: a) A. Baralle, A. Baroudi, M. Daniel, L. Fensterbank, J.-P. Goddard, E. Lacôte, M.-H. Larraufie, G. Maestri, M. Malacria, C. Ollivier in Main-Group Elements in Radical Chemistry - Encyclopedia of Radicals in Chemistry, Biology and Materials (Eds.: C. Chatgilialoglu, A. Studer), John Wiley \& Sons Ltd, Chichester, 2012, vol. 2; b) J. Hartung, T. Gottwald, K. Spehar, Synthesis 2002, 1469-1498.

13 A. Martín, L. M. Quintanal, E. Suárez, Tetrahedron Lett. 2007, 48, 5507-5511.

14 a) O. Mitsunobu, Synthesis 1981, 1-28; b) E. Grochowski, J. Jurczak, Synthesis 1976, 682-684. 
15 For studies of the influence of polar factors on the intermolecular HAT reactions, see: a) A. L. J. Beckwith, A. A. Zavitsas, J. Am. Chem. Soc. 1995, 117, 607-614; b) A. A. Zavitsas, C. Chatgilialoglu, J. Am. Chem. Soc. 1995, 117, 10645-10654; for examples of intramolecular HAT reactions, see: c) C. G. Francisco, R. Freire, A. J. Herrera, I. Pérez-Martín, E. Suárez, Org. Lett. 2002, 4, 1959-1961.

16 a) J.-P. Praly, Adv. Carbohydr. Chem. Biochem. 2001, 56, 65151; b) D. Crich, S. Sun, J. Brunckova, J. Org. Chem. 1996, 61, 605-615; c) N. Yamazaki, E. Eichenberger, D. P. Curran, Tetrahedron Lett. 1994, 35, 6623-6626; d) J. Brunckova, D. Crich, Q. Yao, Tetrahedron Lett. 1994, 35, 6619-6622; e) B. Giese, Radicals in Organic Synthesis: Formation of CarbonCarbon Bonds, Pergamon Press, Oxford, 1986; f) B. Giese, J. Dupuis, Tetrahedron Lett. 1984, 25, 1349-1352; g) J.-P. Praly, Tetrahedron Lett. 1983, 24, 3075-3078.

17 a) A. L. J. Beckwith, S. Brumby, J. Chem. Soc. Perkin Trans. 2 1987, 1801-1807; b) A. L. J. Beckwith, C. J. Easton, J. Am. Chem. Soc. 1981, 103, 615-619; c) K. Hayday, R. D. McKelvey, J. Org. Chem. 1976, 41, 2222-2226.

18 a) C. G. Francisco, A. J. Herrera, A. R. Kennedy, A. Martín, D. Melián, I. Pérez-Martín, L. M. Quintanal, E. Suárez, Chem.
Eur. J. 2008, 14, 10369-10381; b) A. Martín, I. Pérez-Martín, L. M. Quintanal, E. Suárez, J. Org. Chem. 2008, 73, 7710 7720; c) A. Martín, I. Pérez-Martín, L. M. Quintanal, E. Suárez, Org. Lett. 2007, 9, 1785-1788; d) C. G. Francisco, A. J. Herrera, A. R. Kennedy, D. Melián, E. Suárez, Angew. Chem. 2002, 114, 884; Angew. Chem. Int. Ed. 2002, 41, 856-858.

19 D. P. Curran, N. A. Porter, B. Giese, Stereochemistry of Radical Reactions, Wiley-VCH Verlag GmbH, Weinheim, 1996, pp. 131-135.

20 K. Miura, H. Saito, D. Itoh, T. Matsuda, N. Fujisawa, D. Wang, A. Hosomi, J. Org. Chem. 2001, 66, 3348-3355.

21 D. P. Curran, N. A. Porter, B. Giese, Stereochemistry of Radical Reactions, Wiley-VCH Verlag $\mathrm{GmbH}$, Weinheim, 1996, pp. 164-168.

22 D. P. Curran, N. A. Porter, B. Giese, Stereochemistry of Radical Reactions, Wiley-VCH Verlag GmbH, Weinheim, 1996, pp. 119-128.

23 B. Giese, Angew. Chem. 1989, 101, 993; Angew. Chem. Int. Ed. Engl. 1989, 28, 969-980.

Received: March 12, 2012

Published Online: May 31, 2012 